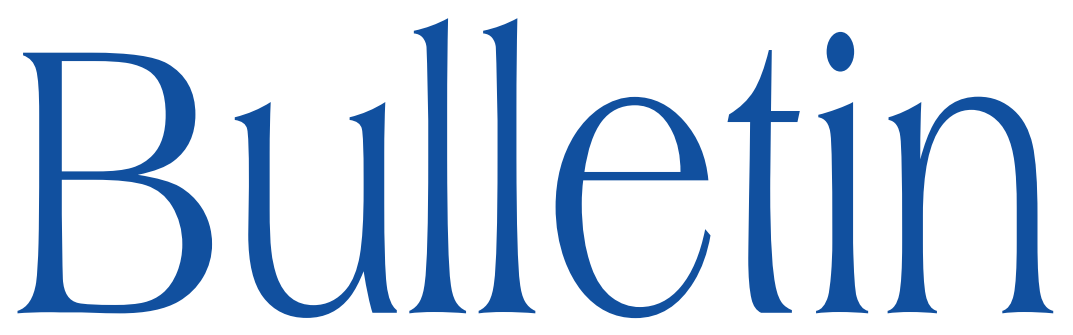

de la SOCIÉTÉ MATHÉMATIQUE DE FRANCE

\title{
ON SOME COMPLETIONS OF THE SPACE \\ OF HAMILTONIAN MAPS
}

\author{
Vincent Humilière
}

\section{Tome 136} Fascicule 3

2008 


\title{
ON SOME COMPLETIONS OF THE SPACE OF HAMILTONIAN MAPS
}

\author{
By VinCENT HuMiLiÈRE
}

\begin{abstract}
In one of his papers, C. Viterbo defined a distance on the set of Hamiltonian diffeomorphisms of $\mathbb{R}^{2 n}$ endowed with the standard symplectic form $\omega_{0}=$ $d p \wedge d q$. We study the completions of this space for the topology induced by Viterbo's distance and some others derived from it, we study their different inclusions and give some of their properties.

In particular, we give a convergence criterion for these distances that allows us to prove that the completions contain non-ordinary elements, as for example, discontinuous Hamiltonians. We also prove that some dynamical aspects of Hamiltonian systems are preserved in the completions.
\end{abstract}

RÉSUmÉ (Sur certains complétés de l'espace des applications hamiltoniennes)

Dans un de ses articles, C. Viterbo définit une distance sur l'ensemble des difféomorphismes hamiltoniens de $\mathbb{R}^{2 n}$, muni de sa forme symplectique standard $\omega_{0}=d p \wedge d q$. Nous étudions les complétés de cet espace pour la topologie induite par la distance de Viterbo, ainsi que d'autres qui en sont dérivées. Nous explicitons leurs différentes inclusions et donnons certaines de leur propriétés.

En particulier, nous donnons un critère de convergence pour ces distances qui nous permet de montrer que les complétés contiennent des éléments intéressants, comme, par exemple, des hamiltoniens discontinus. Nous prouvons aussi que certains aspects de la dynamique hamiltonienne sont préservés dans les complétés.

Texte reçu le 12 janvier 2007, révisé le 8 juin 2007, accepté le 11 janvier 2008

Vincent Humilière, Centre de Mathématiques Laurent Schwartz, UMR 7640 du CNRS, École polytechnique, 91128 Palaiseau, France • E-mail : vincent.humiliere@math.polytechnique.fr 2000 Mathematics Subject Classification. - 53D12, 37J05, 70H20.

Key words and phrases. - Symplectic topology, Hamiltonian dynamics, Viterbo distance, symplectic capacity, Hamilton-Jacobi equation. 


\section{Introduction}

Given an open subset $U$ in $\mathbb{R}^{2 n}$, we denote by $\operatorname{Ham}(U)$ the set of all 1periodic time dependent Hamiltonian functions $\mathbb{R} \times \mathbb{R}^{2 n} \rightarrow \mathbb{R}$ whose support for fixed time is compact and contained in $U$. We will write $\operatorname{Ham}$ for $\operatorname{Ham}\left(\mathbb{R}^{2 n}\right)$.

Given a Hamiltonian function $H \in$ Ham, its symplectic gradient (i.e the unique vector field $X_{H}$ satisfying $\left.d H=\iota_{X_{H}} \omega_{0}\right)$ generates a Hamiltonian isotopy $\left\{\phi_{H}^{t}\right\}$. The set of Hamiltonian diffeomorphisms generated by an element $H$ in $\operatorname{Ham}(U)$ will be denoted by $\mathcal{H}(U)=\left\{\phi_{H}=\phi_{H}^{1} \mid H \in \operatorname{Ham}(U)\right\}$, and we will write $\mathcal{H}$ for $\mathcal{H}\left(\mathbb{R}^{2 n}\right)$. Finally, we call $\mathcal{L}=\left\{\phi\left(0_{n}\right) \mid \phi \in \mathcal{H}\right\}$, the set of Lagrangian submanifolds obtained from the zero section $0_{n} \subset T^{*} \mathbb{R}^{n}=\mathbb{R}^{2 n}$, by a Hamiltonian isotopy with compact support.

As usual, we denote Viterbo's distance on $\mathcal{L}$ or $\mathcal{H}$ by $\gamma$ (see [15]). Convergence with respect to $\gamma$ is called c-convergence.

Our main goals in this paper is to understand the completion $\overline{\mathcal{H}}^{\gamma}$ of the metric space $(\mathcal{H}, \gamma)$, to give some convergence criterion (section 3 ) and to compare it with the convergence for Hofer's distance $d_{H}$ (see [5], chapter 5 section 1).

The notion of $C^{0}$ symplectic topology has been studied by many authors, starting from the work of Eliashberg and Gromov on the $C^{0}$-closure of the group of symplectic diffeomorphisms, to the later results of Viterbo ([15]) and Hofer ([4]).

More recently Oh ([9]) gave a deep study of several versions of $C^{0}$ Hamiltonians. However, our definition seems to differ from his, since in all his definitions, he needs the Hamiltonians to be continuous, while our study starts as we drop this assumption.

Let us now state our main results. For convenience, they will be restated throughout the paper. In section 3 , we introduce a symplectic invariant $\xi_{\infty}$ associated to any subset of $\mathbb{R}^{2 n}$, and prove that

THEOREM 1.1. - Let $\left(H_{k}\right)$ be a sequence of Hamiltonians in Ham, whose supports are contained in a fixed compact set. Suppose there exist a Hamiltonian $H \in$ Ham and a compact set $K \in \mathbb{R}^{2 n}$ with $\xi_{\infty}(K)=0$, such that $\left(H_{k}\right)$ converges uniformly to $H$ on every compact set of $\mathbb{R} \times\left(\mathbb{R}^{2 n}-K\right)$. Then $\left(\phi_{H_{k}}\right)$ converges to $\phi_{H}$ for $\gamma$.

Examples of sets $K$ with $\xi_{\infty}(K)=0$ are given by compact submanifolds of dimension $d \leqslant n-2$.

Viterbo's distance $\gamma$ is defined on $\mathcal{H}$, but we can define for any $H, K \in$ Ham

$$
\gamma_{u}(H, K)=\sup \left\{\gamma\left(\phi_{H}^{t}, \phi_{K}^{t}\right) \mid t \in[0,1]\right\},
$$

TOME $136-2008-\mathrm{N}^{\mathrm{O}} 3$ 
to get a new distance on Ham (we give several variants of this definition). Then the following proposition allows to extend the notion of Hamiltonian flow.

Proposition 1.2. - If we consider the respective completions $\overline{\mathcal{H}}^{\gamma}$ and $\overline{\mathrm{Ham}}^{\gamma}$ of the metric spaces $(\mathcal{H}, \gamma)$ and $\left(\operatorname{Ham}, \gamma_{u}\right)$, then the map $(H, t) \mapsto \phi_{H}^{t}$, Ham $\times$ $\mathbb{R} \rightarrow \mathcal{H}$ induces a map $\overline{\mathrm{Ham}}^{\gamma} \times \mathbb{R} \rightarrow \overline{\mathcal{H}}^{\gamma}$.

The induced map associates to any element $H$ in $\overline{\mathrm{Ham}}^{\gamma}$ a path in $\overline{\mathcal{H}}^{\gamma}$ that we will call the generalized Hamiltonian flow generated by $H$.

We then show that some aspects of Hamiltonian dynamics can be extended to the completions (section 4): We can define a natural action of a generalized flow on a Lagrangian submanifold. We can also associate to it a support and extend the notion of first integral.

To some of them, it is also possible, as we prove in section 6 , to associate a solution to the Hamilton-Jacobi equation:

$$
\frac{\partial u}{\partial t}+H\left(t, x, \frac{\partial u}{\partial x}\right)=0 .
$$

Indeed, a $\gamma_{2}$-Cauchy sequence of Hamiltonians gives a $C^{0}$-Cauchy sequence of solutions (where $\gamma_{2}$ denotes one variant of the distance $\gamma_{u}$ we mentioned above).

In section 5 we give examples of elements in both completions $\overline{\mathcal{H}}^{\gamma}$ and $\overline{\mathrm{Ham}}^{\gamma_{u}}$ that can be described in a much more concrete way than their abstract definition (as equivalence classes of Cauchy sequences). More precisely, we prove

Proposition 1.3. - There is a one-one map

$$
\mathfrak{F}^{\infty} \rightarrow \overline{\mathrm{Ham}}^{\gamma_{u}}
$$

where $\mathfrak{F}^{\infty}$ denotes the set of all functions $H: \mathbb{R} \times \mathbb{R}^{2 n} \rightarrow \mathbb{R} \cup\{+\infty\}$ such that:

(i) $H$ is continuous on $\mathbb{R} \times \mathbb{R}^{2 n}$,

(ii) $H$ vanishes at infinity: $\forall \varepsilon>0, \exists r,(|x|>r \Rightarrow(\forall t,|H(t, x)|<\varepsilon))$,

(iii) there exists a zero capacity set (e.g. an infinitesimally displaceable set), that contains all the points $x$ where $H(t, x)$ is $+\infty$ for all time $t$,

(iv) $H$ is smooth on $\mathbb{R} \times \mathbb{R}^{2 n}-H^{-1}(\{+\infty\})$.

Finally, let us mention that although we developed our theory on $\mathbb{R}^{2 n}$, we can reasonably expect similar results (except those of sections 4.2 and 6) on any compact symplectic manifold satisfying

$$
\left.\omega\right|_{\pi_{2}(M)}=0 \text { and }\left.c_{1}\right|_{\pi_{2}(M)}=0 .
$$

Indeed, on these manifolds, Schwarz defined in [11] a distance which is entirely analogous to Viterbo's. 
Organization of the paper. - In Section 2 we give the definitions of the objects used in the paper. For the reader's convenience, we first recall the construction of Viterbo's distance $\gamma(2.2)$ which is based on the theory of generating functions for Lagrangian submanifolds (2.1). We also remind the reader of the different symplectic capacities constructed from $\gamma(2.3)$. Finally we introduce our new distances derived from $\gamma(2.4)$.

Section 3 is fully devoted to the proof of our convergence criterion. Examples of cases where it holds is then given in 3.3.

In Section 4 we define the completions of Ham and $\mathcal{H}$ and show that some aspects of Hamiltonian dynamics that can be extended to the completions.

In Section 5 we discuss some interesting examples of elements of the completions.

Our results on the Hamilton-Jacobi equation are given in Section 6 .

Finally, we prove in Appendix a "reduction inequality" usefull to prove then all the inequalities between the distances considered in the paper.

Acknowledgments. - I am grateful to my supervisor C. Viterbo for his advices. I also want to thank my friends M. Affre and N. Roy for spending hours correcting my awful English.

\section{Symplectic invariants}

In this section we give the definitions of all the objects we will use in the sequel. We first recall the definition of Viterbo's distance, defined first for Lagrangian submanifolds with the help of generating functions, and then for Hamiltonian diffeomorphisms (see [15]).

2.1. Generating functions quadratic at infinity. - Let $L$ be a Lagrangian submanifold of the cotangent bundle $T^{*} M$ of a smooth manifold $M$. We say that $L$ admits a generating function if there exists an integer $q>0$ and a smooth function $S: M \times \mathbb{R}^{q} \rightarrow \mathbb{R}$ such that $L$ can be written

$$
L=\left\{(x, p) \in T^{*} M \mid \exists \xi \in \mathbb{R}^{q}, \frac{\partial S}{\partial \xi}(x, \xi)=0 \text { and } \frac{\partial S}{\partial x}(x, \xi)=p\right\} .
$$

Such function $S$ is called a generating function quadratic at infinity (or just "g.f.q.i") if there exists a non degenerate quadratic form $Q$ on $\mathbb{R}^{q}$ and a compact $K \subset M \times \mathbb{R}^{q}$ such that, $\forall(x, \xi) \notin K, S(x, \xi)=Q(\xi)$.

For instance, any quadratic form on $\mathbb{R}^{q}$ viewed as a function on $M \times \mathbb{R}^{q}$ is a g.f.q.i of the zero section $0_{M} \subset T^{*} M$. J.C. Sikorav proved in [12] that the property of having a g.f.q.i is invariant by Hamiltonian isotopy with compact support. For this reason we will be interested in the set $\mathcal{L}$ of Lagrangian 
submanifolds, images of the zero section by a Hamiltonian isotopy with compact support.

Furthermore, C. Viterbo and D. Théret proved that the g.f.q.i's of a given Lagrangian submanifold are essentially unique. Before stating this result, let us introduce the following definitions: For a given function $S: M \times \mathbb{R}^{q} \rightarrow \mathbb{R}$, we call a stabilisation of $S$ any function $S^{\prime}: M \times \mathbb{R}^{q} \times \mathbb{R}^{q^{\prime}} \rightarrow \mathbb{R}$ of the form $S^{\prime}\left(x, \xi, \xi^{\prime}\right)=S(x, \xi)+q\left(\xi^{\prime}\right)$, where $q$ is a non-degenerate quadratic form on $\mathbb{R}^{q^{\prime}}$. In addition, two functions $S, S^{\prime}: M \times \mathbb{R}^{q} \rightarrow \mathbb{R}$ are said equivalent if there exists a diffeomorphism $\phi$ of $M \times \mathbb{R}^{q}$ that preserves all the fibers $M \times\{\xi\}$, and a real $C$ such that $S^{\prime}=S \circ \phi+C$.

TheOREm 2.1 ([15, 13]). - Suppose $S, S^{\prime}$ are two g.f.q.i's of the same Lagrangian submanifold in $\mathcal{L}$. Then, up to stabilisation, $S$ and $S^{\prime}$ are equivalent.

This result allows to associate symplectic invariants to any element of $\mathcal{L}$.

\subsection{Invariants defined by minimax and a distance on the group of Hamiltonian dif-}

feomorphisms. - The invariants defined in this section have been introduced by C. Viterbo in [15]. We recall their construction. We first define invariants for Lagrangian submanifolds.

Let $L$ be an element of $\mathcal{L}$ and $S: M \times \mathbb{R}^{q} \rightarrow \mathbb{R}$ be one of its g.f.q.i's. Let us denote $S^{\lambda}=\left\{x \in M \times \mathbb{R}^{q} \mid S(x) \leqslant \lambda\right\}$. Since $S$ is quadratic at infinity, the homotopy types of the pairs $\left(S^{\lambda}, S^{\mu}\right)$ and $\left(S^{\mu}, S^{-\lambda}\right)$ do not depend on $\lambda$, provided that $\lambda$ is sufficiently large . Therefore, we will denote $S^{\infty}$ and $S^{-\infty}$, instead of $S^{\lambda}$ and $S^{-\lambda}$ for $\lambda$ large enough.

Let us introduce $E_{\infty}^{-}$the negative (trivial) bundle of the quadratic form which coincides with $S$ at infinity. We denote $B\left(E_{\infty}^{-}\right), S\left(E_{\infty}^{-}\right)$the ball bundle and the sphere bundle associated to $E_{\infty}^{-}$. The Thom isomorphism is given by $H^{*}(M) \rightarrow H^{*}\left(B\left(E_{\infty}^{-}\right), S\left(E_{\infty}^{-}\right)\right)$, and we also have the isomorphism $H^{*}\left(B\left(E_{\infty}^{-}\right), S\left(E_{\infty}^{-}\right)\right) \simeq H^{*}\left(S^{\infty}, S^{-\infty}\right)$. We will denote by $T$ their composition. For further informations on those isomorphisms, see [6] for example. The inclusion $j_{\lambda}: S^{\lambda} \rightarrow S^{\infty}$ induces a morphism in cohomology $j_{\lambda}^{*}: H^{*}\left(S^{\infty}, S^{-\infty}\right) \rightarrow$ $H^{*}\left(S^{\lambda}, S^{-\infty}\right)$, for all real number $\lambda$. We are now ready for the following

Definition 2.2. - Let $(u, L) \in H^{*}(M) \times \mathcal{L}$, with $u \neq 0$. Using a g.f.q.i $S$ of $L$, we define a real number $c(u, L)$ as follows:

$$
c(u, L)=\inf \left\{\lambda \mid j_{\lambda}^{*} \circ T(u) \in H^{*}\left(S^{\lambda}, S^{-\infty}\right) \text { is non zero }\right\} .
$$

Observe that $c(u, L)$ is well defined, and is independent of the choice of $S$ 's choice, up to additive constant. Indeed, if we replace $S$ with an equivalent or stabilized generating function, the value of $c(u, L)$ does not change, up to additive constant and we conclude using theorem 2.1. Even if it doesn't depend 
on the generating function, we sometimes use the notation $c(u, S)$ instead of $c(u, L)$.

Since the cohomology of the sets $S^{\lambda}$ changes when we cross the level $c(u, L)$, it has to be a critical value of $S$.

Finally, observe that the definition can be extended to classes with compact support $u \in H_{c}^{*}(M)$.

Then, we can use those invariants associated to Lagrangian submanifold to define other invariants associated to Hamiltonian diffeomorphisms.

Consider a Hamiltonian diffeomorphism $\psi \in \mathcal{H}\left(\mathbb{R}^{2 n}\right)$. Its graph $\Gamma_{\psi}$ is a Lagrangian submanifold of $\overline{\mathbb{R}^{2 n}} \times \mathbb{R}^{2 n}\left(=\left(\mathbb{R}^{2 n} \times \mathbb{R}^{2 n},-\omega_{0} \oplus \omega_{0}\right)\right.$, where $\omega_{0}$ is the standard symplectic structure on $\left.\mathbb{R}^{2 n}\right)$. It coincides with the diagonal $\Delta=\left\{(x, x) \mid x \in \mathbb{R}^{2 n}\right\}$, outside the product $B^{2 n}(r) \times B^{2 n}(r)$, for $r$ sufficiently large. When we identify $\overline{\mathbb{R}^{2 n}} \times \mathbb{R}^{2 n}$ with $T^{*} \Delta$ using the map,

$$
(q, p, Q, P) \mapsto\left(\frac{q+Q}{2}, \frac{p+P}{2}, P-p, Q-q\right),
$$

we see that the image $\widetilde{\Gamma_{\psi}}$ of $\Gamma_{\psi}$ is identified with the zero section of $T^{*} \Delta$ outside a compact set.

Then, we can associate the previous invariant to $\widetilde{\Gamma_{\psi}}$ (We normalize generating functions by asking their critical value at infinity to equal 0 ). Let 1 be a generator of $H^{0}\left(\mathbb{R}^{2 n}\right)$ and $\mu$ a generator of $H_{c}^{2 n}\left(\mathbb{R}^{2 n}\right)$.

Definition 2.3 (Viterbo, [15]). — We define,

$$
\begin{gathered}
c_{-}(\psi)=-c\left(\mu, \widetilde{\Gamma_{\psi}}\right), \\
c_{+}(\psi)=-c\left(1, \widetilde{\Gamma_{\psi}}\right), \\
\gamma(\psi)=c_{+}(\psi)-c_{-}(\psi), \\
\gamma(\phi, \psi)=\gamma\left(\psi^{-1} \phi\right) .
\end{gathered}
$$

Let us describe now the properties of the numbers $\gamma, c_{+}$and $c_{-}$that we will use in the paper.

Proposition 2.4 (Viterbo, [15]). - a) (Sign and Separation) For all $\psi$ in $\mathcal{H}$, we have

$$
c_{-}(\psi) \leqslant 0 \leqslant c_{+}(\psi) .
$$

Moreover, $c_{-}(\psi)=c_{+}(\psi)=0$ if and only if $\psi=\mathrm{Id}$.

b) (Triangle inequality) If $\phi$ is another diffeomorphism in $\mathcal{H}$, then

$$
\begin{gathered}
c_{+}(\phi \circ \psi) \leqslant c_{+}(\phi)+c_{+}(\psi), \\
c_{-}(\phi \circ \psi) \geqslant c_{-}(\phi)+c_{-}(\psi), \\
\gamma(\phi \circ \psi) \leqslant \gamma(\phi)+\gamma(\psi) .
\end{gathered}
$$

TOME $136-2008-\mathrm{N}^{\mathrm{O}} 3$ 
In particular, the separation property and the triangle inequality imply that $(\phi, \psi) \rightarrow \gamma(\phi, \psi)$ is a distance on $\mathcal{H}$.

c) (Monotony) Let $\psi_{1}$ and $\psi_{2}$ be two Hamiltonians generated by $H_{1}$ and $H_{2}$. Suppose that for all $(t, x) \in \mathbb{R} \times \mathbb{R}^{2 n}$, we have $H_{1}(t, x) \leqslant H_{2}(t, x)$. Then, $c_{+}\left(\psi_{1}\right) \leqslant c_{+}\left(\psi_{2}\right)$ and $c_{-}\left(\psi_{1}\right) \leqslant c_{-}\left(\psi_{2}\right)$.

As a consequence, if $H$ is a non-negative Hamiltonian, then $c_{-}\left(\phi_{H}\right)=0$. If $H$ is in addition non zero, we deduce $c_{+}\left(\phi_{H}\right)>0$.

d) (Continuity) Let $\mathrm{H}_{1}$ and $\mathrm{H}_{2}$ be two compactly supported hamiltonians, generating $\psi_{1}$ and $\psi_{2}$. Let $\|\cdot\|$ be the usual norm on $C^{0}\left(\mathbb{R}^{2 n} \times[0,1], \mathbb{R}\right)$. If $\left\|H_{1}-H_{2}\right\| \leqslant \varepsilon$, then $\left|\gamma\left(\psi_{1}\right)-\gamma\left(\psi_{2}\right)\right| \leqslant \varepsilon$.

2.3. Two symplectic capacities on $\mathbb{R}^{2 n}$. - We start this section by reminding the reader of the definition of a symplectic capacity. This is a "symplectic" way of measuring sets that plays an important role in symplectic topology. We will use it in particular for our convergence criterion in section 3.

Definition 2.5 (Ekeland-Hofer). - A symplectic capacity on $\left(\mathbb{R}^{2 n}, \omega_{0}\right)$ is a map associating to each subset $U \subset \mathbb{R}^{2 n}$ a number $c(U) \in[0,+\infty]$ satisfying

1. $U \subset V \Rightarrow c(U) \leqslant c(V)$ (monotony),

2. $c(\phi(U))=c(U)$ for all Hamiltonian diffeomorphism $\phi \in \mathcal{H}$ (symplectic invariance),

3. $c(\lambda U)=\lambda^{2} c(U)$ for all real $\lambda>0$ (homogeneity),

4. $c\left(B^{2 n}(1)\right)=c\left(B^{2} \times \mathbb{R}^{2(n-1)}\right)=\pi$, where $B^{2 n}(1)$ is the unit ball of $\mathbb{R}^{2 n}$ (normalisation).

The invariants defined in the previous section allow to define two symplectic capacities as follows ([15]).

Definition 2.6. - 1. For any compact subset $K \subset \mathbb{R}^{2 n}$, we denote by $\gamma(K)$ the number defined by

$$
\gamma(K)=\inf \{\gamma(\phi) \mid \phi(K) \cap K=\varnothing\} .
$$

If $V$ is not compact, we set

$$
\gamma(V)=\sup \{\gamma(K) \mid K \subset V\} .
$$

2. For any open subset $U \subset \mathbb{R}^{2 n}$, we denote by $c(U)$ the number defined by

$$
c(U)=\sup \left\{c_{+}\left(\phi_{H}\right) \mid \operatorname{Supp}(H) \subset U\right\} .
$$

If $V$ is not an open set, we set

$$
c(V)=\inf \{c(U) \mid V \subset U\} .
$$


The maps $c$ and $\gamma$ are symplectic capacities and moreover $c \leqslant \gamma$. We remind the reader of the definition of the displacement energy

$$
d(U)=\inf \left\{d_{H}(\phi, I d) \mid \phi(U) \cap U=\varnothing\right\},
$$

where $d_{H}$ is Hofer's distance defined by

$$
d_{H}(\phi, \psi)=\inf \{\|H-K\| \mid H \text { generates } \phi \text { and } K \text { generates } \psi\},
$$

with $\|H\|=\int_{0}^{1}\left(\max _{x} H(t, x)-\min _{x} H(t, x)\right) d t$.

We are going to define a new symplectic capacity derived from $c$, but before we need the following lemma.

LEMmA 2.7. - We consider a subset $V \subset \mathbb{R}^{2 n}$ and $\mathbb{R}^{2} \times V \subset \mathbb{R}^{2+2 n}$. Then,

$$
c\left(\mathbb{R}^{2} \times V\right) \geqslant c(V) .
$$

That lemma follows from the reduction inequality of Proposition A.1. We postpone its proof to Appendix. The reverse inequality might be true but we are unable to prove it. That leads us to introduce the following object.

Definition 2.8. - For any open subset $U \subset \mathbb{R}^{2 n}$, we set

$$
c^{\infty}(U)=\lim _{N \rightarrow \infty} c\left(U \times \mathbb{R}^{2 N}\right)
$$

and if $V$ is not an open subset,

$$
c^{\infty}(V)=\inf \left\{c^{\infty}(U) \mid V \subset U\right\}
$$

We obtain a symplectic capacity that satisfies $c^{\infty}(V)=c^{\infty}\left(V \times \mathbb{R}^{2}\right)$ for all subset $V$ (this property will be useful), and $c \leqslant c^{\infty}$. Moreover, since $d(U)=$ $d\left(U \times \mathbb{R}^{2 k}\right)$ and $c \leqslant d$, we have $c^{\infty} \leqslant d$. To summarize the known inequalities between capacities we have,

Proposition 2.9. $-c \leqslant \gamma \leqslant d$ and $c \leqslant c^{\infty} \leqslant d$.

2.4. Other distances derived from $\gamma$. - In this section we introduce several other distances for many reason. One is that we want to consider distances not only on $\mathcal{H}$ but also on Ham. Another motivation is our result on the HamiltonJacobi equation (section 6) that needs almost all of them. Finally, a stupid but important reason is that we still don't know which is the best one to develop our theory!

Let us start with the following distance defined on $\mathcal{H}$ already introduced by Cardin and Viterbo in [1].

TOME $136-2008-\mathrm{N}^{\circ} 3$ 
Definition 2.10. - For all Hamiltonian diffeomorphisms $\phi, \psi \in \mathcal{H}$, we define $\tilde{\gamma}(\phi, \psi)=\sup \left\{\gamma\left(\psi^{-1} \phi(L)-L\right) \mid L \in \mathcal{L}\right\}$,

where $\gamma(L)=c(\mu, L)-c(1, L), \forall L \in \mathcal{L}$ and $L_{1}-L_{2}=\left\{\left(q, p_{1}-p_{2}\right) \mid\left(q, p_{1}\right) \in\right.$ $\left.L_{1},\left(q, p_{2}\right) \in L_{2}\right\}$, for $L_{1}, L_{2} \in \mathcal{L}$.

Then, we define distances not anymore on $\mathcal{H}$, but on Ham.

Definition 2.11. - For any $H, K \in$ Ham, we set

$$
\gamma_{u}(H, K)=\sup \left\{\gamma\left(\phi_{H}^{t}, \phi_{K}^{t}\right) \mid t \in[0,1]\right\}
$$

and

$$
\tilde{\gamma}_{u}(H, K)=\sup \left\{\tilde{\gamma}\left(\phi_{H}^{t}, \phi_{K}^{t}\right) \mid t \in[0,1]\right\} .
$$

Here, the subscript "u" means "uniform". Clearly, $\gamma_{u}$ and $\tilde{\gamma}_{u}$ are distances on Ham.

For the next two distances, the principle is to add two dimensions by associating to an Hamiltonian $H$ two suspensions defined on $\mathbb{R} \times \mathbb{R}^{2+2 n}$ :

$$
\begin{gathered}
\hat{H}(s ; t, \tau, x)=\tau+H(t, x), \\
\check{H}(s ; t, \tau, x)=t H(s t, x) .
\end{gathered}
$$

Here, the new time variable is $s$, while the former time variable $t$ becomes a space variable (as a consequence $\hat{H}$ is an autonomous Hamiltonian). We would like to define our distances by $\hat{\gamma}(H, K)=\gamma(\hat{H}, \hat{K})$ and $\check{\gamma}(H, K)=\gamma(\check{H}, \check{K})$. But since $\hat{H}$ and $\check{H}$ are not compactly supported we have to be slightly more subtle.

Definition 2.12. - Let $\rho$ be a fixed real function defined on $[0,+\infty)$, supposed to be non-negative, smooth, decreasing, with support in [0,1], flat at 0 and such that $\rho(0)=1$. For every natural integer $\alpha$ and every real number $t$, we set $\rho_{\alpha}(t)=1$ if $-\alpha \leqslant t \leqslant \alpha$, and $\rho_{\alpha}(t)=\rho(|t|-\alpha)$ otherwise.

We denote by $\hat{H}_{\alpha}$ and $\check{H}_{\alpha}$ the Hamiltonian functions defined on $\mathbb{R} \times \mathbb{R}^{2+2 n}$, by

$$
\hat{H}_{\alpha}(s ; t, \tau, x)=\rho_{\alpha}(\tau) \tau+\rho_{\alpha}(t) H(t, x),
$$

and

$$
\check{H}_{\alpha}(s ; t, \tau, x)=\rho_{\alpha}(t) t H(s t, x) .
$$

Then, for $H, K \in$ Ham, we set

$$
\hat{\gamma}(H, K)=\limsup _{\alpha \rightarrow+\infty} \gamma_{u}\left(\hat{H}_{\alpha}, \hat{K}_{\alpha}\right)
$$

and

$$
\check{\gamma}(H, K)=\limsup _{\alpha \rightarrow+\infty} \gamma\left(\phi_{\check{H}_{\alpha}}, \phi_{\check{K}_{\alpha}}\right) .
$$


Remark that $\hat{\gamma}(H, K)$ and $\check{\gamma}(H, K)$ are finite. Indeed, if we denote by $B$ a ball containing both supports of $H$ and $K$, then $\hat{H}_{\alpha}, \hat{K}_{\alpha}, \check{H}_{\alpha}$ and $\check{K}_{\alpha}$ have support in $\mathbb{R}^{2} \times B$, for any integer $\alpha$. Hence $\gamma\left(\phi_{\check{H}_{\alpha}}, \phi_{\check{K}_{\alpha}}\right) \leqslant 2 c\left(\mathbb{R}^{2} \times B\right) \leqslant 2 c^{\infty}(B)$ (See section 2.3 for notations). It shows that the lim sup in the definition of $\check{\gamma}$ is finite. The same proof shows that $\hat{\gamma}(H, K)$ is also finite.

The triangle inequality for $\hat{\gamma}$ and $\check{\gamma}$ is a direct consequence of the triangle inequality for $\gamma$. The separation property is obtained from the separation property for $\gamma$ and Proposition 2.13.

For convenience, we will not write the subscript $\alpha$ anymore. In the following, we will denote $\hat{H}$ for $\hat{H}_{\alpha}$, and $\check{H}$ for $\check{H}_{\alpha}$.

REMARKS. - By repeating these constructions several times (i.e., by taking suspensions of suspensions), we can construct new distances. For example, we will use in section 6 the distance $\gamma_{2}=\lim \sup _{\alpha \rightarrow+\infty} \check{\gamma}\left(\hat{H}_{\alpha}, \hat{K}_{\alpha}\right)$.

Using the invariance of $\gamma$, it is easy to verify that the suspended distances $\hat{\gamma}$, $\check{\gamma}$ and $\gamma_{2}$ are invariant under the action of $\mathcal{H}$. Namely, for $H, K$ Hamiltonians and $\varphi$ Hamiltonian diffeomorphism, we have:

$$
\begin{aligned}
\hat{\gamma}(H \circ \varphi, K \circ \varphi) & =\hat{\gamma}(H, K), \\
\check{\gamma}(H \circ \varphi, K \circ \varphi) & =\check{\gamma}(H, K), \\
\gamma_{2}(H \circ \varphi, K \circ \varphi) & =\gamma_{2}(H, K) .
\end{aligned}
$$

The following proposition gives inequalities between the distances. It will be proved in Appendix. It is based on the reduction inequality (Proposition A.1).

Proposition 2.13. - We have $\tilde{\gamma} \leqslant \gamma$ and $\tilde{\gamma}_{u} \leqslant \gamma_{u} \leqslant \min (\hat{\gamma}, \check{\gamma})$.

\section{The convergence criterion}

This is the central section of our paper. We give there the proof of our main result, Theorem 1.1. 


\subsection{A sufficient condition for a Hamiltonian diffeomorphism to be $\gamma$-close to $I d$}

We start this section with some formulas concerning Hamiltonian flows. They can be obtained by direct computation (see [5], page 144).

Lemma 3.1. - For all Hamiltonians $H$ and $K$, with compact support, we have:

$$
\begin{gathered}
\phi_{\bar{H}}^{t}=\left(\phi_{H}^{t}\right)^{-1}, \quad \text { where } \bar{H}(t, x)=-H\left(t, \phi^{t}(x)\right) \\
\phi_{H \# K}^{t}=\phi_{H}^{t} \circ \phi_{K}^{t}, \text { where }(H \# K)(t, x)=H(t, x)+K\left(t,\left(\phi^{t}\right)^{-1}(x)\right) \\
\phi_{\overline{H \# K}}^{t}=\left(\phi_{H}^{t}\right)^{-1} \circ \phi_{K}^{t} .
\end{gathered}
$$

REMARK. - $(\bar{H} \# K)(t, x)=(K-H)\left(t, \phi^{t}(x)\right)$.

The following proposition shows that if a sequence of Hamiltonians $\left(H_{n}\right)$ converges to zero uniformly on every compact set contained in the complement of a set whose capacity is zero, then $\left(\phi_{H_{n}}\right)$ converges to $I d$ for $\gamma$.

Proposition 3.2. - Let $H$ be a Hamiltonian on $\mathbb{R}^{2 n}$ with compact support. If $U$ is an open subset of $\mathbb{R}^{2 n}$, such that $c(U) \leqslant \varepsilon$ and $|H(t, x)| \leqslant \varepsilon$ for all $t \in[0,1]$ and all $x \in \mathbb{R}^{2 n}-U$, then $\gamma\left(\phi_{H}\right) \leqslant 4 \varepsilon$.

Proof. - Let $K_{1}, K_{2}$ be Hamiltonians with compact support, such that $0 \leqslant$ $K_{i} \leqslant 1, i=1,2, K_{1}$ equals 1 on the support of $H$ and $K_{2}$ equals 1 on the support of $K_{1}$ (hence $K_{1} \leqslant K_{2}$ ). Denote $\psi_{1, \varepsilon}$ the diffeomorphism generated by $H-\varepsilon K_{1}$, and $\psi_{2, \varepsilon}$ the diffeomorphism generated by $\varepsilon K_{2}$. Then we have $H \leqslant \varepsilon K_{2}+\left(H-\varepsilon K_{1}\right)$. As $\left(\psi_{2, \varepsilon}\right)^{-1}$ coincides with $I d$ on the support of $H-\varepsilon K_{1}$, the lemma 3.1 implies that $\varepsilon K_{2}+H-\varepsilon K_{1}$ is the Hamiltonian that generates $\psi_{2, \varepsilon} \circ \psi_{1, \varepsilon}$. The monotony, the triangle inequality and the continuity (Proposition 2.4) then give

$$
c_{+}(\phi) \leqslant c_{+}\left(\psi_{2, \varepsilon} \circ \psi_{1, \varepsilon}\right) \leqslant c_{+}\left(\psi_{2, \varepsilon}\right)+c_{+}\left(\psi_{1, \varepsilon}\right) \leqslant \varepsilon+c_{+}\left(\psi_{1, \varepsilon}\right) .
$$

Denote by $\widetilde{\psi_{1, \varepsilon}}$ the diffeomorphism generated by a non-negative Hamiltonian, with support in $U$, and greater than $H-\varepsilon K_{1}$. Then by the monotony property, $c_{+}\left(\psi_{1, \varepsilon}\right) \leqslant c_{+}\left(\widetilde{\psi_{1, \varepsilon}}\right)$. Finally, since $\operatorname{Supp}\left(\widetilde{\psi_{1, \varepsilon}}\right) \subset U$, we get $c_{+}\left(\widetilde{\psi_{1, \varepsilon}}\right) \leqslant c(U) \leqslant$ $\varepsilon$.

Using the inequality $H \geqslant-\varepsilon K_{2}+\left(H+\varepsilon K_{1}\right)$, we obtain the same type of inequality for $c_{-}$.

For example, if $K$ is a compact submanifold of dimension lower or equal than $n-1$, then $d(K)=0$ (and hence $c(K)=0)$. 


\subsection{What about non-identity elements that are close for $\gamma$ ?}

Unfortunately, the previous result cannot be straightforwardly generalised to obtain a general convergence criterions when the limit is not zero. Indeed, we can find two Hamiltonians that are $C^{0}$-close out of a null-capacity set, but not $\gamma$-close.

Example. - It is well known that the capacities $c$ and $\gamma$ of the unit sphere $\mathcal{S}=\left\{x \in \mathbb{R}^{2 n} \mid\|x\|=1\right\}$ are $\pi$. It is also true for $c^{\infty}$. Then, for all $\varepsilon>0$, there exists a Hamiltonian $H$ with support in a small neighbourhood $U$ of $\mathcal{S}$, and such that $c_{+}\left(\phi_{H}\right)>\pi-\varepsilon$. Because of the monotony property (proposition??), $H$ can be chosen non-negative. We set $U^{+}$a neighbourhood of $\left\{x \in \mathcal{S} \mid x_{1} \geqslant 0\right\}$ and $U^{-}$a neighbourhood of $\left\{x \in \mathcal{S} \mid x_{1}<0\right\}$, such that $U=U^{+} \cup U^{-}$. If $U$, $U^{+}$and $U^{-}$are choosen small enough, we have $d\left(U^{ \pm}\right)<\varepsilon$ and by proposition $2.9 c^{\infty}\left(U^{ \pm}\right)<\varepsilon$. Using some partition of unity associated to the decomposition $U=U^{+} \cup U^{-}$, we get two functions $H^{ \pm}$, with support in $U^{ \pm}$and such that $H=H^{+}+H^{-}$.

Now, we see that $H^{+}$coincides with $H$ outside $U^{-}$, whose capacity verifies $c^{\infty}\left(U^{ \pm}\right)<\varepsilon$, but on the other hand,

$$
\left\|\gamma\left(\phi_{H}\right)-\gamma\left(\phi_{H^{+}}\right)\right\| \geqslant \gamma\left(\phi_{H}, \phi_{H^{+}}\right) \geqslant \pi-\varepsilon-\gamma\left(\phi_{H^{-}}\right) \geqslant \pi-2 \varepsilon .
$$

It shows that the previous statement is false when the limit is not zero.

Nevertheless, we can introduce a new invariant, in order to extend the result of proposition 3.2 .

Definition 3.3. - For any subset $U$ and any Hamiltonian $H \in$ Ham, we define

$$
\xi^{H}(U)=c^{\infty}\left(\bigcup_{t \in[0,1]} \phi_{H}^{t}(U)\right) .
$$

We may then set

$$
\xi_{\lambda}(U)=\sup \xi^{H}(U), \text { for } 0<\lambda \leqslant \infty,
$$

where the supremum is over all Hamiltonian functions $H$ with $\gamma_{u}(H) \leqslant \lambda$.

THEOREM 3.4. - Let $H_{1}$ and $H_{2}$ be Hamiltonians on $\mathbb{R}^{2 n}$ with compact support. Let $U$ be a subset of $\mathbb{R}^{2 n}$, satisfying one of the two following conditions:

1. $\xi_{\infty}(U) \leqslant \varepsilon$.

2. $\exists \lambda>0, \xi_{\lambda}(U)=0$

If $\left|H_{1}(t, x)-H_{2}(t, x)\right| \leqslant \varepsilon$ for all $t \in[0,1]$ and all $x \notin U$, then we have $\gamma\left(\phi_{H_{1}}, \phi_{H_{2}}\right) \leqslant 4 \varepsilon$. 
Proof. - Consider the Hamiltonian $H(t, x)=H_{1}\left(t, \phi_{2}^{t}(x)\right)-H_{2}\left(t, \phi_{2}^{t}(x)\right)$. By assumption, $|H(t, x)| \leqslant \varepsilon$, for all $(t, x)$ with $x \notin \phi_{2}^{-t}(U)$ and hence for all $t$ and all $x \notin \bigcup_{t \in[0,1]}\left(\phi_{2}^{-1}\right)^{t}(U)$. Each condition on $U$ implies

$$
c\left(\bigcup_{t \in[0,1]}\left(\phi_{2}^{-1}\right)^{t}(U)\right) \leqslant c^{\infty}\left(\bigcup_{t \in[0,1]}\left(\phi_{2}^{-1}\right)^{t}(U)\right) \leqslant \varepsilon .
$$

By proposition 3.2 and lemma 3.1 , we get $\gamma\left(\phi_{H_{1}}, \phi_{H_{2}}\right)=\gamma\left(\phi_{H}\right) \leqslant 4 \varepsilon$.

Important remark. - In the proof of theorem 3.4, we see that the important condition is in fact $\xi^{H_{2}}(U) \leqslant \varepsilon$, which is of course implied by both conditions $\xi_{\infty}(U) \leqslant \varepsilon$ and $\xi_{\lambda}(U)=0$.

COROLlaRY 3.5. - The conclusion of theorem 3.4 still holds if we replace $\gamma$ with $\tilde{\gamma}$. For the distances on Ham, we get under the same assumptions

$$
\mathfrak{d}\left(H_{1}, H_{2}\right) \leqslant 4\left\|H_{1}-H_{2}\right\|_{C^{0}}
$$

where $\mathfrak{d}$ is either $\tilde{\gamma_{u}}, \gamma_{u}, \hat{\gamma}$ or $\check{\gamma}$.

Proof. - By proposition 2.13 (inequality between distances), we just have to prove it for $\hat{\gamma}$ and $\check{\gamma}$. Then remark that under the hypothesis of theorem 3.4, we have $\left|\hat{H}_{1}(s ; t, \tau, x)-\hat{H}_{2}(s ; t, \tau, x)\right| \leqslant \varepsilon$ and $\left|\check{H}_{1}(s ; t, \tau, x)-\check{H}_{2}(s ; t, \tau, x)\right| \leqslant \varepsilon$ for all integer $\alpha$, all $s \in[0,1]$, and all $(t, \tau, x) \notin \mathbb{R}^{2} \times U$.

Unfortunately, even if $U$ satisfies one of the conditions of proposition 3.4, it is not in general the case for $\mathbb{R}^{2} \times U$. However, by the above remark, it is sufficient to show that for all real number $\delta>0$ and all integer $\alpha$ large enough, $\xi^{\breve{H}_{2}}\left(\mathbb{R}^{2} \times U\right) \leqslant \varepsilon+\delta$ and $\xi^{\hat{H}_{2}}\left(\mathbb{R}^{2} \times U\right) \leqslant \varepsilon+\delta$. By letting $\delta$ tend to zero and taking limsup with respect to $\alpha$, we obtain $\hat{\gamma}(H, K) \leqslant 4 \varepsilon$ and $\check{\gamma}(H, K) \leqslant 4 \varepsilon$ as required.

Let us denote $F$ for $\check{H}_{2}$ or $\hat{H}_{2}$. The inequalities on $\xi^{F}$ come directly from the expression of $\phi_{\breve{H}_{2}}$ and $\phi_{\hat{H}_{2}}$ (see computations in Appendix A.2). Indeed, in both cases,

$$
\bigcup_{s \in[0,1]}\left(\phi_{F}^{-1}\right)^{s}\left([-\alpha, \alpha]^{2} \times U\right) \subset \mathbb{R}^{2} \times \bigcup_{s \in[0,1]}\left(\psi^{-1}\right)^{s}(U),
$$

where $\psi^{s}$ is a Hamiltonian isotopy that appears in last coordinate when we compute $\phi_{F}$. Therefore, since $\xi^{F}\left(\mathbb{R}^{2} \times U\right)=\lim _{\alpha \rightarrow+\infty} \xi^{F}\left([-\alpha, \alpha]^{2} \times U\right)$, we 
get for any $\delta>0$ and any $\alpha$ large enough:

$$
\begin{aligned}
\xi^{F}\left(\mathbb{R}^{2} \times U\right) & \leqslant \delta+c^{\infty}\left(\mathbb{R}^{2} \times \bigcup_{s \in[0,1]}\left(\psi^{-1}\right)^{s}(U)\right) \\
& =\delta+c^{\infty}\left(\bigcup_{s \in[0,1]}\left(\psi^{-1}\right)^{s}(U)\right) \leqslant \delta+\varepsilon .
\end{aligned}
$$

That concludes the proof.

Corollary 3.6. - Let $\left(H_{k}\right)$ be a sequence of Hamiltonians in Ham, whose supports are contained in a fixed compact set. Suppose there exist a Hamiltonian $H \in \mathrm{Ham}$ and a compact set $K \in \mathbb{R}^{2 n}$ with $\xi_{\infty}(K)=0$, such that $\left(H_{k}\right)$ converges uniformly to $H$ on every compact set of $\mathbb{R} \times\left(\mathbb{R}^{2 n}-K\right)$. Then $\left(\phi_{H_{k}}\right)$ converges to $\phi_{H}$ for $\tilde{\gamma}, \gamma$, and $\left(H_{k}\right)$ converges to $H$ for $\tilde{\gamma_{u}}, \gamma_{u}, \hat{\gamma}, \check{\gamma}$.

Proof. - For $\tilde{\gamma}, \gamma$, it is a direct consequence of the remark that follows theorem 3.4. We just have to verify that for all $\varepsilon>0$, there exists a small neighbourhood $U$ of $K$ such that $\xi^{H}(U) \leqslant \varepsilon$. This is true because for every neighbourhood $V$ of $\bigcup_{t \in[0,1]} \phi_{H}(K)$, we can choose a neighbourhood $U$ of $K$ such that

$$
\bigcup_{t \in[0,1]} \phi_{H}(U) \subset V .
$$

Since $c^{\infty}\left(\bigcup_{t \in[0,1]} \phi_{H}(K)\right)=0$ and $\bigcup_{t \in[0,1]} \phi_{H}(K)$ is compact, we can choose $V$ such that $c^{\infty}(V) \leqslant \varepsilon$, and obtain $c^{\infty}\left(\bigcup_{t \in[0,1]} \phi_{H}(U)\right) \leqslant \varepsilon$ as required.

For $\hat{\gamma}$ and $\check{\gamma}$, we have to verify that for all $\varepsilon>0$ and all $\delta>0$, there exists a small neighbourhood $U$ of $K$ such that for all $\alpha$ large enough $\xi^{\phi}(U) \leqslant \varepsilon+\delta$, where $F$ is either $\hat{H}$ or $\check{H}$. The proof made above for $\phi_{H}$ shows that we can find $U$ such that $\xi^{f}(U) \leqslant \varepsilon$, where $f$ generates the isotopy $\psi^{s}$ defined as in the proof of corollary 3.5. Therefore we have for all $\delta$ and all $\alpha$ large enough, $\xi^{F}\left(\mathbb{R}^{2} \times U\right) \leqslant \xi^{F}\left([-\alpha, \alpha]^{2} \times U\right)+\delta \leqslant \xi^{f}(U)+\delta \leqslant \varepsilon+\delta$.

By proposition 2.13, corollary 3.6 is also true for $\tilde{\gamma_{u}}$ and $\gamma_{u}$.

REMARK. - Similar proofs give that theorem 3.4 and corollary 3.6 still hold for $\gamma_{2}$.

\subsection{Example of a non trivial $\xi$-small set}

Proposition 3.7. - Let $U$ be a closed submanifold of $\mathbb{R}^{2 n}$ whose dimension $d$ verifies $d \leqslant n-2$. Then $\xi_{\infty}(U)=0$. 
Proof. - Let $H \in$ Ham. The problem is that $\bigcup_{t \in[0,1]} \phi_{H}^{t}(U)$ is not in general a manifold. To avoid that problem, we are going to add two dimensions and make a suspension in this way. We denote by $\Phi$ the Hamiltonian diffeomorphism on $\mathbb{R}^{2+2 n}=\{(t, \tau, x)\}$ generated by the Hamiltonian

$$
[0,1] \times \mathbb{R}^{2+2 n} \rightarrow \mathbb{R},(s ; t, \tau, x) \mapsto t H(t s, x) .
$$

We also set $V=\Phi([0,1] \times[-1,1] \times U)$. The computation of $\Phi$ gives

$$
\Phi(t, \tau, x)=\left(t, \tau-H(t, x), \phi^{t}(x)\right) .
$$

We see that $\bigcup_{t \in[0,1]} \phi_{H}^{t}(U)$ can be obtained from $V$ by symplectic reduction by the coisotropic manifold $\{\tau=0\}$. So we are going to look for a Hamiltonian diffeomorphism $\phi_{K}$ that displaces $V$ and preserves $\{\tau=0\}$ at the same time. If the Hamiltonian does not depend on $t$, the second condition is verified. Since $V$ is compact, it is sufficient for $K$ to verify

$$
\forall v \in V, \mathbb{R} X_{K}(v) \cap T_{v} V=\{0\},
$$

which is equivalent to

$$
\forall v \in V, \operatorname{ker} d K(v) \oplus T_{v} V^{\perp}=\mathbb{R}^{2+2 n}
$$

and to

$$
\forall v \in V, T_{v} V^{\perp} \not \subset \operatorname{ker} d K(v) .
$$

That makes us consider the 1 -jet bundle $J^{1}\left(\mathbb{R} \times \mathbb{R}^{1+2 n}, \mathbb{R}\right)$ and its submanifold

$$
W=\left\{(s, q ; \sigma, p ; z) \mid(s, q) \in V, z \in \mathbb{R}, T_{(s, q)} V^{\perp} \subset \operatorname{ker}(\sigma, p)\right\} .
$$

The dimension of $W$ is exactly $2 n+1$. Indeed, the vector space $\{(\sigma, p) \in$ $\left.\mathbb{R}^{2 n+2^{*}} \mid T_{(s, q)} V^{\perp} \subset \operatorname{ker}(\sigma, p)\right\}$ has dimension $2 n+2-\operatorname{dim}\left(T_{(s, q)} V^{\perp}\right)=n$.

By Thom transversality theorem (see [3] for example), there exists a function $L$ whose 1 -jet verifies $j^{1} L \pitchfork W$. But $j^{1} L$ can be seen as a function $\mathbb{R} \times \mathbb{R}^{1+2 n} \rightarrow$ $J^{1}\left(\mathbb{R} \times \mathbb{R}^{1+2 n}, \mathbb{R}\right)$, and by lemma 4.6 page 53 in [3], we have for a generic choice of $s \in \mathbb{R}, j^{1} L(s, \cdot) \pitchfork W$. We fix $s$ as previously and we denote $K: \mathbb{R}^{2+2 n} \rightarrow \mathbb{R}$, $K(t, \cdot)=L(s, \cdot)$

Then, notice that for every $s, q, p, z$, the set of all $\sigma$ such that $(s, q ; \sigma, p ; z) \in$ $W$ is either $\varnothing$ or $\mathbb{R}$. It can be shown by direct computation of $T V^{\perp}$, whose first component appears to be always $\{0\}$. As a consequence, we get $j^{1} K \pitchfork W\left(j^{1} K\right.$ differs from $j^{1} L(s, \cdot)$ just by its $\sigma$-component which is $\{0\}$ instead of $\frac{\partial L}{\partial s}(s, \cdot)$ for $\left.j^{1} L(s, \cdot)\right)$.

Now, since $(2 n+2)+(2 n+1)=\operatorname{dim}\left(j^{1} K\left(\mathbb{R}^{2+2 n}\right)\right)+\operatorname{dim}(W)<\operatorname{dim}\left(J^{1}(\mathbb{R} \times\right.$ $\left.\left.\mathbb{R}^{1+2 n}, \mathbb{R}\right)\right)=4 n+5$, we get $j^{1} K\left(\mathbb{R}^{2+2 n}\right) \cap W=\varnothing$. It follows that $K$ satisfies the two conditions: it preserves $\{\tau=0\}$ and it satisfies

$$
\forall v \in V, \mathbb{R} X_{K}(v) \cap T_{v} V=\{0\} .
$$


As $V$ is compact, for $\varepsilon$ small enough, since $\phi_{\varepsilon K}=\phi_{K}^{\varepsilon}$, we have $\phi_{\varepsilon K}(V) \cap V=\varnothing$. In addition $\varepsilon K$ can be made as $C^{0}$-small as we want.

We are now ready for the reduction by $\{\tau=0\}$. Since it preserves $\{\tau=0\}$, $\varepsilon K$ induces a Hamiltonian on the reduction $\mathbb{R}^{2 n}$. This Hamiltonian is $C^{0}$-small and generates a diffeomorphism $\psi$ whose Hofer's distance to identity $d_{H}(\psi, i d)$ is small, and that satisfies

$$
\psi\left(\bigcup_{t \in[0,1]} \phi_{H}^{t}(U)\right) \cap \bigcup_{t \in[0,1]} \phi_{H}^{t}(U)=\varnothing .
$$

This Hamiltonian is not compactly supported, but any Hamiltonian with compact support which coincides with it on a sufficiently large ball, would have the same properties. That proves $d\left(\bigcup_{t \in[0,1]} \phi_{H}^{t}(U)\right)=0$, and since $c^{\infty} \leqslant d$, we get $\xi^{H}(U)=0$.

\section{Completions and extension of Hamiltonian dynamics}

In this section, we introduce the completions and give the first properties of their elements: the existence of a flow that acts on Lagrangian submanifolds, the notion of first integral and the existence of a support. The full section 6 will be devoted to another property related to the Hamilton-Jacobi equation.

4.1. Notations, inclusions and definitions. - Let us denote respectively $\overline{\mathcal{H}}^{\gamma}$, $\overline{\operatorname{Ham}}^{\gamma}, \overline{\mathcal{H}}^{\tilde{\gamma}}, \overline{\operatorname{Ham}}^{\tilde{\gamma}}{ }^{\prime}, \overline{\mathrm{Ham}}^{\hat{\gamma}}, \overline{\mathrm{Ham}}^{\check{\gamma}}$ and $\overline{\mathrm{Ham}}^{\gamma}$ the completions of $(\mathcal{H}, \gamma)$, $\left(\operatorname{Ham}, \gamma_{u}\right),(\mathcal{H}, \tilde{\gamma}),\left(\operatorname{Ham}, \tilde{\gamma}_{u}\right),(\operatorname{Ham}, \hat{\gamma}),(\operatorname{Ham}, \check{\gamma})$ and $\left(\operatorname{Ham}, \gamma_{2}\right)$.

The sets $\overline{\mathcal{H}}^{\gamma}$ and $\overline{\mathcal{H}}^{\tilde{\gamma}}$ have a natural structure of group with bi-invariant metric induced by the natural structures on $(\mathcal{H}, \gamma)$ and $(\mathcal{H}, \tilde{\gamma})$. Moreover we have the following fact:

Proposition 4.1. - The map $H \mapsto \phi_{H}^{1}$ induces Lipschitz maps $\overline{\mathrm{Ham}}^{\gamma_{u}} \rightarrow$ $\overline{\mathcal{H}}^{\gamma}$ and $\overline{\operatorname{Ham}}^{\tilde{\gamma}} \rightarrow \overline{\mathcal{H}}^{\tilde{\gamma}}$.

Proof. - Indeed, by construction of the distances, $H \mapsto \phi_{H}^{1}$ is Lipschitz both as a map $\left(\operatorname{Ham}, \gamma_{u}\right) \rightarrow(\mathcal{H}, \gamma)$ and as a map $\left(\operatorname{Ham}, \tilde{\gamma}_{u}\right) \rightarrow(\mathcal{H}, \tilde{\gamma})$.

The inequalities between the different distances, proved in Proposition 2.13, induce inclusions between the completions which may be summarized by the following diagram. Here, $\overline{\mathcal{H}}^{d_{H}}$ denotes the completion of $\mathcal{H}$ for Hofer's distance $d_{H}$ (which satisfies $d_{H} \leqslant \gamma$ ) and $C_{c}$ the set of continuous (not necessarily smooth) Hamiltonians with compact support. 


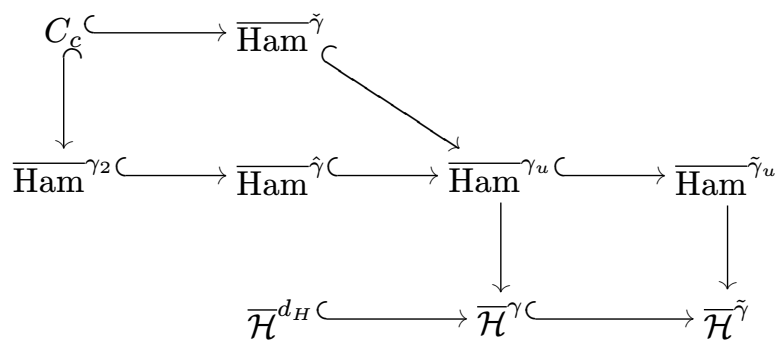

As in Proposition 4.1, the map $(H, t) \mapsto \phi_{H}^{t}$, Ham $\times \mathbb{R} \rightarrow \mathcal{H}$ induces maps $\overline{\mathrm{Ham}}^{\gamma_{u}} \times \mathbb{R} \rightarrow \overline{\mathcal{H}}^{\gamma}$ and $\overline{\mathrm{Ham}}^{\tilde{\gamma}_{u}} \times \mathbb{R} \rightarrow \overline{\mathcal{H}}^{\tilde{\gamma}}$. Therefore, any element $H$ in one of the completions $\overline{\mathrm{Ham}}^{\gamma}$, $\overline{\mathrm{Ham}}^{\tilde{\gamma}}$, $\overline{\mathrm{Ham}}^{\hat{\gamma}}, \overline{\mathrm{Ham}}^{\check{\gamma}}$ or $\overline{\mathrm{Ham}}^{\gamma_{2}}$ can be associated a path in either $\overline{\mathcal{H}}^{\gamma}$, or $\overline{\mathcal{H}}^{\tilde{\gamma}}$. This path of course has the semi-group property. That leads us to the following definition.

Definition 4.2. - Such a path will be called the generalized Hamiltonian flow generated by $H$.

4.2. Action on Lagrangian submanifolds. - Recall that the set $\mathcal{L}$ of Lagrangian submanifolds isotopic to the zero section by compactly supported Hamiltonian isotopy, can be endowed with Viterbo's distance, also denoted $\gamma$ (set $\gamma\left(L_{1}, L_{2}\right)=\gamma\left(L_{1}-L_{2}\right)$, see definition 2.10). Let us denote $\mathfrak{L}$ the completion of $\mathcal{L}$ with respect to this distance.

Proposition 4.3. - The groups $\overline{\mathcal{H}}^{\gamma}$ and $\overline{\mathcal{H}}^{\tilde{\gamma}}$ naturally act on the set $\mathfrak{L}$. This action extends the natural action of $\mathcal{H}$ on $\mathcal{L}$.

Proof. - It is a simple consequence of the inequality $\tilde{\gamma} \leqslant \gamma$ (Proposition 2.13 proved in Appendix).

Let $L \in \mathfrak{L}$ represented by a sequence $\left(L_{k}\right)$ and $\phi$ in $\overline{\mathcal{H}}^{\gamma}$ (the proof is the same for $\left.\overline{\mathcal{H}}^{\tilde{\gamma}}\right)$, represented by a sequence $\left(\phi_{k}\right)$. We are going to show that $\left(\phi_{k}\left(L_{k}\right)\right)$ defines an element of $\mathfrak{L}$ that we will denote $\phi(L)$.

This follows easily from the fact that for $\phi, \psi \in \mathcal{H}$ and $L, M \in \mathcal{L}$,

$$
\begin{aligned}
\gamma(\phi(L)-\psi(M)) & \leqslant \gamma(\phi(L)-\psi(L))+\gamma(\psi(L)-\psi(K)) \\
& \leqslant \gamma\left(\psi^{-1} \phi(L)-L\right)+\gamma(L-K) \\
& \leqslant \tilde{\gamma}(\phi, \psi)+\gamma(L-K) .
\end{aligned}
$$


REMARK. - A consequence of Proposition 4.3 is that we can define what is a Lagrangian submanifold invariant under a generalized flow.

That leads us to another question which is: Can we define what is an invariant hypersurface of a generalized flow?

A (partial) answer to this question is that we can define what is a first integral of a generalized Hamiltonian flow.

4.3. Notion of first integral. - This property has been first mentioned in [1], in the definition (3.3) of the so-called $c$-commuting Hamiltonians. Let us restate it with our notations.

An element in one of the completions $\overline{\mathrm{Ham}}^{\gamma}, \overline{\mathrm{Ham}}^{\tilde{\gamma}}{ }_{u}, \overline{\mathrm{Ham}}^{\hat{\gamma}}, \overline{\mathrm{Ham}}^{\check{\gamma}}$ and $\overline{\mathrm{Ham}}^{\gamma}$ will be said autonomous if it can be represented by a Cauchy sequence of time-independent Hamiltonian functions.

Definition 4.4. - Let $H, K$ be two elements in one of the above completions, generating two respective generalized flows $\phi_{H}^{t}$ and $\phi_{K}^{t}$. Then we will say that $H$ and $K$ commute, or that $K$ is a first integral of $H$ if $\phi_{K}^{s} \phi_{H}^{t} \phi_{K}^{-s} \phi_{H}^{-t}=I d$.

In other words, $K$ is a first integral of $H$ if there exists two Cauchy sequences $\left(H_{n}\right)$ and $\left(K_{n}\right)$ representing $H$ and $K$, such that for all $s$ and $t, \phi_{K_{n}}^{s} \phi_{H_{n}}^{t} \phi_{K_{n}}^{-s} \phi_{H_{n}}^{-t}$ c-converges to $I d$.

It is proved in [1] that this definition extends the usual definition of commuting Hamiltonian functions.

4.4. Existence of a support. - In this section, we state a lemma which makes it possible to define a support for the elements of the different completions.

LEMMA 4.5. - a. Let $\left(\phi_{n}\right)$ be a sequence in $\mathcal{H}$ converging to a Hamiltonian diffeomorphism $\phi$, with respect to $\gamma$ or $\tilde{\gamma}$. Assume that there exists a set $U \in \mathbb{R}^{2 n}$ such that $\operatorname{supp}\left(\phi_{n}\right) \subset U$. Then $\operatorname{supp}(\phi) \subset \bar{U}$.

b. Let $\left(H_{n}\right)$ be a sequence in Ham converging to a smooth Hamiltonian function $H$, with respect to $\gamma_{u}, \tilde{\gamma}_{u}, \hat{\gamma}, \check{\gamma}$, etc. Assume that there exists a set $U \in \mathbb{R}^{2 n}$ such that $\operatorname{supp}\left(H_{n}\right) \subset U$. Then $\operatorname{supp}(H) \subset \bar{U}$.

Proof. - a. Thanks to Proposition 2.13, we just have to prove the assertion in the case of $\tilde{\gamma}$. Suppose $\operatorname{supp}(\phi) \not \subset \bar{U}$. Then there exists an $x$ in $\mathbb{R}^{2 n}-\bar{U}$ such that $\phi(x) \neq x$. Let $\psi$ be a Hamiltonian diffeomorphism whose support is included in $\mathbb{R}^{2 n}-\bar{U}$ and which does not contain $\phi(x)$. Suppose in addition that $\psi(x) \neq x$. Then, since the supports of $\phi_{n}$ and $\psi$ are disjoint, we have $\psi \circ \phi_{n}^{-1} \circ \psi^{-1} \circ \phi_{n}=I d$, for all integer $n$. Taking limit, we get on one hand $\psi \circ \phi^{-1} \circ \psi^{-1} \circ \phi=I d$. But on the other hand, we have by construction, $\psi \circ \phi^{-1} \circ \psi^{-1} \circ \phi(x)=\psi(x) \neq x$, which is contradictory. 
b. We use the first part of the lemma to conclude that for all time $t$, $\operatorname{supp}\left(\phi^{t}\right) \subset \bar{U}$. This implies that $\operatorname{supp}(H) \subset \bar{U}$.

REMARK. - A similar argument shows that the property of letting globally invariant any sphere centered at 0 , is invariant by taking $\gamma$ or $\tilde{\gamma}$ limits. Similarly, a $\gamma_{u}, \tilde{\gamma}_{u}, \hat{\gamma}$ or $\check{\gamma}$ limit of radial Hamiltonians is radial.

Definition 4.6. - a. Let $\psi$ be an element of $\overline{\mathcal{H}}^{\gamma}$ or $\overline{\mathcal{H}}^{\tilde{\gamma}}$. Then we define $\operatorname{support}(\psi)$ as

$\bigcap\left\{\bar{U} \mid U\right.$ open set, such that there exists $\left(\psi_{n}\right)$ representing $\psi$ such that

$$
\left.\forall n, \operatorname{supp}\left(\psi_{n}\right) \subset U\right\},
$$

where "supp" denotes the usual notions of support for Hamiltonian diffeomorphisms.

b. Let $K$ be an element of $\overline{\operatorname{Ham}}^{\gamma}, \overline{\operatorname{Ham}}^{\gamma_{u}}, \overline{\operatorname{Ham}}^{\hat{\gamma}}, \overline{\mathrm{Ham}}^{\check{\gamma}}$ or $\overline{\mathrm{Ham}}^{\gamma}$. Then we define $\operatorname{support}(K)$ as

$\bigcap\left\{\bar{U} \mid U\right.$ open set, such that there exists $\left(K_{n}\right)$ representing $K$ such that

$$
\left.\forall n, \operatorname{supp}\left(K_{n}\right) \subset U\right\},
$$

where "supp" denotes the usual notions of support for smooth Hamiltonians.

These new notions of support coincide with the usual notions for smooth Hamiltonians and Hamiltonian diffeomorphisms. Indeed, let $\eta$ be either a Hamiltonian diffeomorphism viewed as an element of $\overline{\mathcal{H}}^{\gamma}$ or $\overline{\mathcal{H}}^{\tilde{\gamma}}$, or a smooth Hamiltonian seen as an element of $\overline{\mathrm{Ham}}^{\gamma_{u}}, \overline{\mathrm{Ham}}^{\tilde{\gamma}}$, $\overline{\mathrm{Ham}}^{\hat{\gamma}}, \overline{\mathrm{Ham}}^{\check{\gamma}}$ or $\overline{\mathrm{Ham}}^{\gamma_{2}}$. Let $\left(\eta_{n}\right)$ be a sequence representing $\eta$, and $U$ an open set with $\operatorname{supp}\left(\eta_{n}\right) \subset U$ for all $n$. Then lemma 4.5 gives $\operatorname{supp}(\eta) \subset \bar{U}$. Hence $\operatorname{supp}(\eta) \subset \operatorname{support}(\eta)$. Conversely, for any neighbourhood $U$ of $\operatorname{supp}(\eta)$ the constant sequence $(\eta)$ converges to $\eta$ and has support in $U$. Therefore $\operatorname{support}(\eta) \subset \bigcap_{\mathcal{V}} \bar{U}$, where the intersection is over the set $\mathcal{V}$ of all open neighbourhoods of $\operatorname{supp}(\eta)$. Then, it is easy to see that $\bigcap_{\mathcal{V}} \bar{U}=\bigcap_{\mathcal{V}} U=\operatorname{supp}(\eta)$.

\section{Description of some elements of the completions}

The elements of the different completions are by definition equivalence classes of Cauchy sequences. So they are defined in a very abstract way. In this section, we show that many elements of the completions can be seen in a more concrete way. 
5.1. Examples in the completion of Ham. - The inequalities between Hofer's distance and our four distances $\gamma_{u}, \tilde{\gamma}_{u}, \hat{\gamma}$ and $\check{\gamma}$ on Ham imply inclusions of the completions. In particular any continuous time-dependent Hamiltonian can be seen as an element of $\overline{\mathrm{Ham}}^{\gamma_{u}}, \overline{\mathrm{Ham}}^{\tilde{\gamma}_{u}}, \overline{\mathrm{Ham}}^{\hat{\gamma}}$ and $\overline{\mathrm{Ham}}^{\check{\gamma}}$.

In view of Theorem 3.4 and Corollary 3.6, we can conjecture that if $K \in \mathbb{R}^{2 n}$ satisfies $\xi^{\infty}(K)=0$ a sequence $\left(H_{k}\right)$ converges uniformly on compact sets of $\mathbb{R} \times\left(\mathbb{R}^{2 n}-K\right)$ to a function $H$ continuous on $\mathbb{R} \times\left(\mathbb{R}^{2 n}-K\right)$, then $\left(H_{k}\right)$ is Cauchy for either $\gamma_{u}, \tilde{\gamma}_{u}, \hat{\gamma}$ or $\check{\gamma}$ (compare with Corollary 3.6). We are still unable to prove it, but if we restrict to a family of Hamiltonians which converge to $+\infty$ at their discontinuity points, this result can be established.

Definition 5.1. - We denote by $\mathfrak{F}$ the set of all functions $H: \mathbb{R} \times \mathbb{R}^{2 n} \rightarrow$ $\mathbb{R} \cup\{+\infty\}$ such that:

(i) There exists $K \in \mathbb{R}^{2 n}$ with $c^{\infty}(K)=0$ such that $H(t, x)=+\infty \Rightarrow x \in K$,

(ii) $H$ vanishes at infinity: $\forall \varepsilon>0, \exists r,(|x|>r \Rightarrow(\forall t,|H(t, x)|<\varepsilon))$,

(iii) $H$ is continuous on $\mathbb{R} \times \mathbb{R}^{2 n}$.

We also set $\mathfrak{F}^{\infty}=\left\{H \in \mathfrak{F} \mid H\right.$ is smooth on $\left.\mathbb{R} \times \mathbb{R}^{2 n}-H^{-1}(\{+\infty\})\right\}$, and $\mathfrak{A}$, $\mathfrak{A}^{\infty}$ the subsets of time-independent elements of $\mathfrak{F}$ and $\mathfrak{F}^{\infty}$.

For the elements of $\mathfrak{F}^{\infty}$, the set of discontinuity is somehow "stable" under the Hamiltonian flow. This property allows to consider functions with a larger discontinuity set than what could be expected in the general case $\left(c^{\infty}(K)=0\right.$ instead of $\left.\xi^{\infty}(K)=0\right)$.

Lemma 5.2. - Suppose $H$ is an element of $\mathfrak{A}$ and $K=H^{-1}(\{+\infty\})$. Then there exists a sequence of smooth autonomous Hamiltonians $\left(H_{k}\right) \in$ Ham with the following properties:

a. $\left(H_{k}\right)$ converges to $H$ uniformly on every compact subset of $\mathbb{R}^{2 n}-K$.

b. $\left(H_{k}\right)$ is Cauchy for $\gamma_{u}, \tilde{\gamma}_{u}, \hat{\gamma}$ and $\check{\gamma}$.

Moreover, if $H \in \mathfrak{A}^{\infty}$, then any sequence $\left(H_{k}\right)$ that converges to $H$ uniformly on the compact subsets of $\mathbb{R}^{2 n}-K$, does not converge in Ham, for none of the distances $\gamma_{u}, \tilde{\gamma}_{u}, \hat{\gamma}$ and $\check{\gamma}$.

Proof. - Fix $k>0$. Properties (ii) and (iii) in Definition 5.1 imply that $K$ is compact. Since $c^{\infty}(K)=0$, there exists an open neighborhood $U$ of $K$ such that $c^{\infty}(U) \leqslant \frac{1}{k}$. Then, if we denote $H^{>A}=\{x \mid H(x)>A\}$, we have for $A$ large enough, $K \subset H^{>A} \subset U$. Indeed, if it was not true, then for all integer for all integer $l>0$, there would exists a point $a_{l}$ in $H^{>l}$, but not in $U$. Then, the sequence $\left(a_{l}\right)$ would take values in $H^{\geqslant 1} \cap\left(\mathbb{R}^{2 n}-U\right)$ which is compact, and hence it would have a subsequence that would converge to an element of $K \cap\left(\mathbb{R}^{2 n}-U\right)$, which contradicts our assumption. Let us fix a real number $A_{k}$ such that $H^{>A_{k}} \subset U$. 
Now, let $H_{k}$ be a smooth function with compact support such that $\mid H_{k}-$ $H \mid<\frac{1}{k}$ on $\mathbb{R}^{2 n}-H^{>A_{k}+\frac{2}{k}}$, and such that $\left|H_{k}-A_{k}-\frac{2}{k}\right|<\frac{1}{k}$ on $H^{>A_{k}+\frac{2}{k}}$. The sequence $\left(H_{k}\right)$ clearly converges to $H$ uniformly on every compact subset of $\mathbb{R}^{2 n}-K$. Let us see why it is Cauchy.

By Proposition 2.13, we just have to prove it for $\hat{\gamma}$ and $\check{\gamma}$. We write $F_{k}$ for either $\breve{H}_{k}$ or $\hat{H}_{k}$. We also denote, as in the proof of Corollary $3.5, \psi_{k}$ for the third coordinate of $\phi_{F_{k}}$. Since $H_{k}$ is an autonomous Hamiltonian, its flow $\phi_{H_{k}}^{t}$ preserves its level sets. Hence, the isotopy $\psi_{k}^{s}$ preserves the level sets of $F_{k}$ (see the computations in Appendix A.2). Therefore, since by construction $H^{>A_{k}+\frac{2}{k}} \subset H_{k}^{>A_{k}+\frac{1}{k}}$, we have

$$
\bigcup_{t \in[0,1]} \psi_{k}^{t}\left(H^{>A_{k}+\frac{2}{k}}\right) \subset H_{k}^{>A_{k}+\frac{1}{k}}
$$

Let $\delta>0$ and suppose $\alpha$ is sufficiently large. Then, as in the proof of Corollary 3.5 ,

$$
\begin{aligned}
\xi^{F_{k}}\left(\mathbb{R}^{2} \times H^{>A_{k}+\frac{2}{k}}\right) & \leqslant \delta+c^{\infty}\left(\mathbb{R}^{2} \times \bigcup_{s \in[0,1]}\left(\psi_{k}^{-1}\right)^{s}\left(H^{>A_{k}+\frac{2}{k}}\right)\right) \\
& \leqslant \delta+c^{\infty}\left(\bigcup_{s \in[0,1]}\left(\psi_{k}^{-1}\right)^{s}\left(H^{>A_{k}+\frac{2}{k}}\right)\right) \\
& \leqslant \delta+c^{\infty}\left(H_{k}^{>A_{k}+\frac{1}{k}}\right) .
\end{aligned}
$$

Since $H_{k}^{>A_{k}+\frac{1}{k}} \subset H^{>A_{k}} \subset U$ and $c^{\infty}(U) \leqslant \frac{1}{k}$, we obtain $\xi^{F_{k}}\left(H^{>A_{k}+\frac{2}{k}}\right) \leqslant \frac{1}{k}+\delta$.

Now, pick an integer $l \geqslant k$. If $l$ and $k$ are large enough, then we have $\left|\hat{H}_{k}-\hat{H}_{l}\right| \leqslant \frac{1}{k}$ and $\left|\check{H}_{k}-\check{H}_{l}\right| \leqslant \frac{1}{k}$ on $\mathbb{R}^{2+2 n}-\left(\mathbb{R}^{2} \times H^{>A_{k}+\frac{2}{k}}\right)$. Therefore, by the remark that follows Theorem 3.4, we get $\hat{\gamma}\left(H_{l}, H_{k}\right) \leqslant \frac{4}{k}$ and $\check{\gamma}\left(H_{l}, H_{k}\right) \leqslant \frac{4}{k}$, after taking limsup with respect to $\alpha$. It proves that $\left(H_{k}\right)$ is a Cauchy sequence for $\tilde{\gamma}_{u}, \gamma_{u}, \hat{\gamma}$, and $\check{\gamma}$.

Suppose now that $H$ is smooth on $\mathbb{R}^{2 n}-K$. Then we can choose $H_{k}$ such that it coincides with $H$ on $B_{k}-H^{>A_{k}+\frac{2}{k}}$, where $B_{k}$ is the ball of radius $k$, centered at 0 . Suppose that $\left(H_{k}\right)$ converges to a Hamiltonian $L \in$ Ham for $\tilde{\gamma}_{u}$, $\gamma_{u}, \hat{\gamma}$, and $\check{\gamma}$. Then for any integer $k, \overline{H_{k}} \# H_{l}$ converges to $\overline{H_{k}} \# L$ while $l$ tends to infinity for $\tilde{\gamma}_{u}$ (see Lemma 3.1 for notations). According to Lemma 4.5, since $\overline{H_{K}} \# H_{l}$ has support in the complementary of $B_{k}-H^{>A_{k}+\frac{2}{k}}, \overline{H_{k}} \# L$ has support in its closure and hence $L$ coincides with $H$ on $B_{k}-H^{>A_{k}+\frac{2}{k}}$. Since it is true for any $k, L$ has to coincide with $H$ on $\mathbb{R}^{2 n}-K$. Therefore $L$ cannot belong to Ham, which contradicts our assumptions.

Finally, if $\left(L_{k}\right)$ is another sequence of Hamiltonians that converges to $H$ uniformly on the compact subsets of $\mathbb{R}^{2 n}-K$, then, similarly as in the above proof that $\left(H_{k}\right)$ is Cauchy, we obtain that $\hat{\gamma}\left(L_{k}, H_{k}\right)$ and $\check{\gamma}\left(L_{k}, H_{k}\right)$ converge 
to 0 , where $H_{k}$ is the particular sequence defined in the previous paragraph. Since $\left(H_{k}\right)$ does not converge, $\left(L_{k}\right)$ does not converge either.

REMARK. - As usual, the results of Lemma 5.2 still hold for $\gamma_{2}$.

Proposition 5.3. - The set $\mathfrak{F}^{\infty}$ can be embedded into each completion $\overline{\operatorname{Ham}}^{\gamma}, \overline{\operatorname{Ham}}^{\tilde{\gamma}}$, $\overline{\operatorname{Ham}}^{\hat{\gamma}}$ and $\overline{\mathrm{Ham}}^{\tilde{\gamma}}$.

Proof. - Let us first consider the autonomous case (elements of $\mathfrak{A}^{\infty}$ ).

Since $\overline{\mathrm{Ham}}^{\hat{\gamma}} \subset \overline{\mathrm{Ham}}^{\gamma_{u}} \subset \overline{\mathrm{Ham}}^{\tilde{\gamma}_{u}}$ and $\overline{\mathrm{Ham}}^{\check{\gamma}} \subset \overline{\mathrm{Ham}}^{\gamma_{u}} \subset \overline{\mathrm{Ham}}^{\tilde{\gamma} u}$, it is enough to prove it for $\hat{\gamma}$ and $\check{\gamma}$. We will make the proof for $\hat{\gamma}$ and the proof for $\check{\gamma}$ will be exactly the same. Let $J$ be the function that associates to any $H \in \mathfrak{A}^{\infty}$ the element of $\overline{\mathrm{Ham}}^{\hat{\gamma}}$ represented by any sequence $\left(H_{k}\right)$ that converges uniformly to $H$ on the compact sets of $\mathbb{R}^{2 n}-H^{-1}(\{+\infty\})$. As we noticed at the end of the proof of Lemma 5.2, two such sequences are equivalent and hence $J$ is well-defined.

Let us now prove that $J$ is one-one. Let $H, G \in \mathfrak{A}^{\infty}$ and let $\left(H_{k}\right),\left(G_{k}\right)$ be two sequences respectively associated to them, precisely constructed as in the last but one paragraph of the previous proof. Suppose that $G \neq H$, we are going to show that $\gamma\left(H_{k}, G_{k}\right)$ does not converge to zero, that will imply that $\hat{\gamma}\left(H_{k}, G_{k}\right)$ does not converge to zero.

We can define almost everywhere the flows $\phi_{G}^{t}, \phi_{H}^{t}$ and $\psi^{t}=\phi_{G}^{-t} \circ \phi_{H}^{t}$. Let $\psi_{k}=\phi_{G_{k}}^{-1} \circ \phi_{H_{k}}$. Since $G \neq H$, there exists a point $x$ such that $\psi(x) \neq x$. Hence, there exists a small ball $B$ around $x$ such that $\psi(B) \cap B=\varnothing$. Let $K$ be a compact neighborhood of $\bigcup_{t} \psi^{t}(B)$. For $k$ large enough, $H_{k}$ and $G_{k}$ coincide respectively with $H$ and $G$ on $K$, and thus $\psi_{k}(B) \cap B=\varnothing$ too. Since $\gamma\left(H_{k}, G_{k}\right)=\gamma\left(\psi_{k}\right) \geqslant \gamma(B)>0, \gamma\left(H_{k}, G_{k}\right)$ cannot converge to zero.

To achieve the proof, we just have to notice that the map $H \mapsto \hat{H}$ is a oneone map $\mathfrak{F}_{2 n}^{\infty} \rightarrow \mathfrak{A}_{2 n+2}^{\infty}$ (the subscript denotes the dimension of the ambient symplectic space). Thus, according to the autonomous case, if $H$ is in $\mathfrak{F}_{2 n}^{\infty}$ then $\hat{H}$ is in $\overline{\mathrm{Ham}}^{\gamma_{u}}\left(\mathbb{R}^{2 n+2}\right)$. Moreover, according to Lemma 5.2, we may construct a Cauchy sequence $H_{k}$ of the form $\hat{F}_{k}$ for some Hamiltonians $F_{k}$. That means that $H$ is actually an element of $\overline{\operatorname{Ham}}^{\hat{\gamma}}\left(\mathbb{R}^{2 n}\right)$. Inclusions between completions give that it is an element of $\overline{\operatorname{Ham}}^{\gamma}\left(\mathbb{R}^{2 n}\right)$ and $\overline{\operatorname{Ham}}^{\tilde{\gamma}}\left(\mathbb{R}^{2 n}\right)$ too. finally, a similar reasoning using $\gamma_{2}$ instead of $\hat{\gamma}$ allows to see $H$ as an element of $\overline{\operatorname{Ham}}^{\tilde{\gamma}}\left(\mathbb{R}^{2 n}\right)$. 
Now, if we denote by $C_{0}$ the set of continuous Hamiltonians that vanish at infinity, we can improve the diagram of section 4.1:

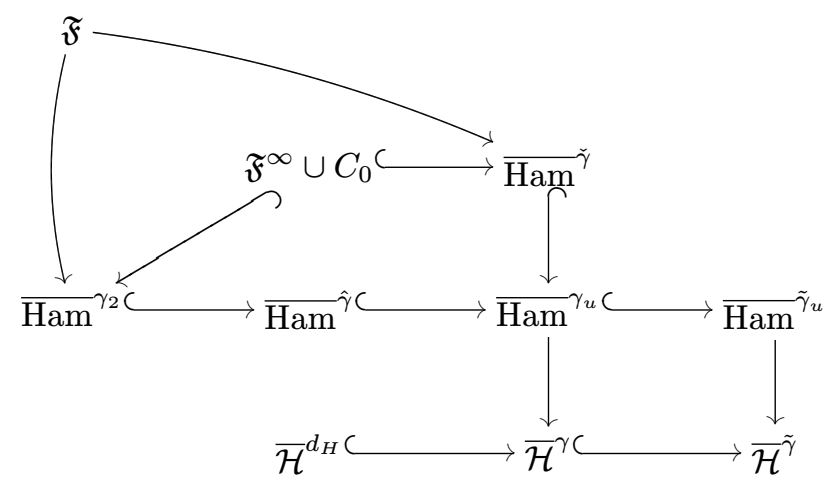

5.2. Examples in the completions of $\mathcal{H}$. - In the completions of Ham easy examples was given by continuous Hamiltonian functions. In the completions of $\mathcal{H}$ there are no similar result. Indeed, there are no known relation between $C^{0}$-distance and $\gamma$.

However, we can give concrete examples of elements of the completion of $\mathcal{H}$ by Proposition 5.3. Indeed, it implies that the (generalized) flows generated by elements of $\mathfrak{F}^{\infty}$ are in both $\overline{\mathcal{H}}^{\gamma}$ and $\overline{\mathcal{H}}^{\tilde{\gamma}}$. Let us give some examples (in their construction, $\gamma$ can be replaced by $\tilde{\gamma}$ without any problem).

Example of a non smooth homeomorphism in $\overline{\mathcal{H}}^{\gamma}$. - We consider a decreasing function $h:[0,+\infty) \rightarrow[0,+\infty)$, with support in $[0,1]$, and equal to 1 on $[0,3 / 4]$. Then we define $H_{k}(x)=\sum_{i=1}^{k} h\left(2^{i}|x|^{2}\right)$, for $x \in \mathbb{R}^{2}$ and $H(x)=$ $\sum_{i=1}^{\infty} h\left(2^{i}|x|^{2}\right)$ (the "sky-scrapper" Hamiltonian). Let us see why $\phi_{H}$ can be seen as a non-smooth homeomorphism.

Lemma 4.5 implies that $\phi_{H}$ coincides with $\phi_{H_{k}}$ out of $B_{2^{-k}}$. So, we can compute the explicit form of $\phi$. In polar coordinates, we obtain:

$$
\phi_{H}(r, \theta)=\left(\theta-r^{2} f^{\prime}\left(r^{2}\right), r\right)
$$

for $r>0$ where $f(s)=\sum_{i>0} h\left(2^{i} s\right.$ ) (for any $s$, all the terms in this sum are 0 except maybe one). We see that $\phi_{H}$ is a homeomorphism. It is moreover a fibered rotation whose rotation number has no limit at 0 . Thus $\phi_{H}$ is not smooth.

Example of a discontinuous element in $\overline{\mathcal{H}}^{\gamma}$. - In the previous example, the sequence of diffeomorphisms $\left(\phi_{H_{k}}\right)$ was converging almost everywhere to a homeomorphism (which was not a diffeomorphism). Therefore, one could think that 
the class of $\left(\phi_{H_{k}}\right)$ in the completion $\overline{\mathcal{H}}^{\gamma}$ can be represented by a homeomorphism. However, with the help of Proposition 5.2, we can show that it is not true in general, at least in dimension $2 n \geqslant 4$.

Indeed, consider $H: \mathbb{R}^{2} \times \mathbb{R}^{2 n} \rightarrow \mathbb{R}$,

$$
\left(x_{1}, x_{2}\right) \mapsto \frac{1}{\left|\left\|x_{1}\right\|^{2}-1\right|+\left\|x_{2}\right\|^{2}} \chi\left(\left\|\left(x_{1}, x_{2}\right)\right\|\right),
$$

where $\chi$ is smooth with compact support and equals 1 on the ball of radius 2 centered at zero. Clearly, $H \in \mathfrak{F}^{\infty}$ (because $K=H^{-1}(\{+\infty\})=\mathbb{S}^{1} \times\{0\}$ satisfies $c^{\infty}(K)=0$ as required). Consider the sequence $\left(H_{k}\right)$ constructed in the proof of Lemma 5.2. Since $\left(H_{k}\right)$ is Cauchy for $\gamma_{u},\left(\phi_{H_{k}}\right)$ is Cauchy for $\gamma$. Suppose it converges to an element $\phi$. Then, Lemma 4.5 implies that for any neighbourhood $U$ of $K$ and for $k$ large enough, $\phi$ coincides with $\phi_{H_{k}}$ on $\mathbb{R}^{2+2 n}-U$. Therefore, we can compute the explicit form of $\phi$ on $\mathbb{R}^{2+2 n}-K$.

In polar coordinates $\left(s_{1}, \theta_{1}, s_{2}, \theta_{2}\right)$ with $s_{1}=\left\|x_{1}\right\|^{2}$ and $s_{2}=\left\|x_{2}\right\|^{2}$, we get for $s_{1}<1$ :

$$
\phi\left(s_{1}, 0,0,0\right)=\left(s_{1}, \frac{s_{1}}{\left(1-s_{1}\right)^{2}}, 0,0\right) .
$$

If we let $s_{1}$ converge to 1 , we see that $\phi$ is not continuous.

Questions. - The previous examples lead us to natural questions: Are all the elements of $\overline{\mathcal{H}}^{\gamma}\left(\mathbb{R}^{2}\right)$ homeomorphisms? Conversely, can we see any symplectic homeomorphism (element of the $C^{0}$-closure of symplectic diffeomorphisms in the homeomorphisms in general dimension, area-preserving homeomorphisms in dimension 2) as an element of $\overline{\mathcal{H}}^{\gamma}$ ?

This last question is related to Oh's still open question whether his group of "Hamiltonian homeomorphisms", called Hameo, equals or not the group of symplectic homeomorphisms [9].

\section{Application to the Hamilton-Jacobi equation}

Let $H$ be a smooth Hamiltonian function on $\mathbb{R} \times \mathbb{R}^{2 n}$. We consider the evolution Hamilton-Jacobi equation $(H J)$ :

$$
\frac{\partial u}{\partial t}+H\left(t, x, \frac{\partial u}{\partial x}\right)=0,
$$

where $u: \mathbb{R} \times \mathbb{R}^{n} \rightarrow \mathbb{R},(t, x) \mapsto u(t, x)$ satisfies an initial condition $u(0, x)=$ $u_{0}(x)$. First, we remind the reader of the construction of a variational solution of $(H J)$ (see for example [14] or [10]). 
6.1. Recall on variational solutions of $(H J)$. - Let us denote by $\Lambda_{0}$ the graph of $d u_{0}$ and call it the initial submanifold. In fact, the following construction can be made for any Lagrangian submanifold $\Lambda_{0} \subset \mathbb{R}^{2 n}$. We consider $\Sigma=$ $\hat{H}^{-1}(\{0\}) \subset \mathbb{R}^{2+2 n}$. A geometric solution of $(H J)$ is a Lagrangian submanifold $L$ that satisfies $\Lambda_{0} \leqslant L \leqslant \Sigma$. For example, the graph of the differential of a smooth function $u$ is a geometric solution if and only if $u$ itself is a solution of $(H J)$.

With the help of the flow $\phi_{\hat{H}}^{t}$, we can construct a geometrical solution $L_{H}=$ $\bigcup_{t \in I} \phi_{\hat{H}}^{t}\left(\Lambda_{0}\right)$, where $I$ is an open interval containing $[0,1]$ and such that $\rho_{\alpha}=1$ on $I$. The Lagrangian submanifold $L_{H}$ obtained is an element of $\mathcal{L}\left(\mathbb{R}^{2+2 n}\right)$.

For any element $L \in \mathcal{L}\left(\mathbb{R}^{2 k}\right)$, we can associate a function $u_{L}$ on $\mathbb{R}^{2 k}$ by the following method.

Let $S: \mathbb{R}^{k} \times \mathbb{R}^{q} \rightarrow \mathbb{R}$ be a g.f.q.i of $L$. Denote by $1_{z}$ the fundamental class in $H^{0}(z)$. Then, we define $u_{L}$ by

$$
u_{L}(z)=c\left(1_{z},\left.S\right|_{z \times \mathbb{R}^{q}}\right),
$$

with notations of section 2. The function $u_{L}$ is everywhere $C^{0}$, and it is proved in [10], that $u_{L}$ is $C^{k}$ on a dense open set, for $k \geqslant 1$. Moreover, when it is defined, we have $\left(x, d u_{L}(x)\right) \in L$. Therefore, the function $u_{L_{H}}$ is a solution of $(H J)$ on any open set on which it is smooth.

We are now going to prove an interesting property of the elements of $\overline{\mathrm{Ham}}^{\gamma_{2}}$, which is the fact that we can extend to them the construction of a variational solution of $(H J)$.

\subsection{Extension to the completion}

Proposition 6.1. - Let $H$ and $K$ be two Hamiltonian functions, and $u_{L_{H}}$, $u_{L_{K}}$ the solution obtained by the above method with the same initial submanifold $\Lambda_{0}$. Then,

$$
\left\|u_{L_{H}}-u_{L_{K}}\right\|_{C^{0}} \leqslant \gamma_{2}(H, K) .
$$

That leads us to the following definition.

Definition 6.2. - Let $H \in \overline{\mathrm{Ham}}^{\gamma}$. A continuous function $u$ will be called generalized variational solution of $(H J)$ for $H$, if there exists a Cauchy sequence $\left(H_{k}\right)$ in Ham representing $H$ and such that the sequence of solutions $\left(u_{L_{H_{k}}}\right)$ $C^{0}$-converges to $u$.

Therefore, proposition 6.1 implies the following statement:

Theorem 6.3. - For each initial condition $u_{0}$, any element $H$ in the completion $\overline{\mathrm{Ham}}^{\gamma_{2}}$ admits a unique generalized variational solution $u_{H}$. Moreover, the so constructed map $\overline{\mathrm{Ham}}^{\gamma_{2}} \rightarrow C^{0}$ is continuous. 
In particular, any Hamiltonian function in $\mathfrak{F}^{\infty}$ (see definition 5.1) admits a unique generalized variational solution.

Proof. - Let $\left(H_{k}\right) \in$ Ham be a Cauchy sequence for $\gamma_{2}$ representing an element $H \in \overline{\mathrm{Ham}}^{\gamma}$. Then, proposition 6.1 implies that $\left(u_{L_{H_{k}}}\right)$ is a Cauchy sequence in $C^{0}$ and hence converges to a continuous function $u$. Moreover, if $\left(H_{k}\right)$ and $\left(F_{k}\right)$ are two equivalent Cauchy sequences for $\gamma_{2}$, then proposition 6.1 also implies that $\left(u_{L_{H_{k}}}\right)$ and $\left(u_{L_{F_{k}}}\right)$ are equivalent, and hence converge to the same limit. It gives the existence and the unicity.

The continuity of the map $\overline{\mathrm{Ham}}^{\gamma} \rightarrow C^{0}$ is also an immediate consequence of Proposition 6.1.

To prove proposition 6.1 , we first prove the following lemma:

Lemma 6.4. - For any $L \in \mathcal{L}$, we have

$$
\left\|u_{L}\right\|_{C^{0}} \leqslant \gamma(L)
$$

Proof. - Since $L$ coincides with the zero section out of a compact set, $u_{L}$ has a compact support. It follows that $\left\|u_{L}\right\|_{C^{0}} \leqslant \max \left(u_{L}\right)-\min \left(u_{L}\right)$. We will prove that $\min \left(u_{L}\right) \geqslant c(1, L)$. It will also imply that $\max \left(u_{L}\right) \leqslant c(\mu, L)$ by Poincaré duality. Indeed, using the fact that $c(\mu, L)=-c(1, \bar{L})$ and that for all $z, \mu_{z}=1_{z}$, we have $u_{L}=-u_{\bar{L}}$.

Let $z \in \mathbb{R}^{k}$, and $S: \mathbb{R}^{k} \times \mathbb{R}^{q} \rightarrow \mathbb{R}$ be a g.f.q.i of $L \subset \mathbb{R}^{2 k}$. Then, $\left.S\right|_{\{z\} \times \mathbb{R}^{q}}$ is a g.f.q.i. of the reduction of $L$ by the coisotropic submanifold $\{z\} \times \mathbb{R}^{k} \subset \mathbb{R}^{2 k}$. Therefore, by lemma A.2, we get $c\left(1_{z},\left.S\right|_{\{z\} \times \mathbb{R}^{q}}\right) \geqslant c(1, S)$, for all $z$ and hence $\min \left(u_{L}\right) \geqslant c(1, L)$ as required.

Proof of proposition 6.1. - The proposition comes from a sequence of inequalities:

$$
\left\|u_{L_{H}}-u_{L_{K}}\right\|_{C^{0}} \leqslant \gamma\left(L_{H}, L_{K}\right) \leqslant \tilde{\gamma}\left(\phi_{\tilde{\hat{H}}}, \phi_{\tilde{\hat{K}}}\right) \leqslant \gamma_{2}(H, K) .
$$

The third inequality comes from the first inequality in proposition 2.13. The second one is proved in [1]. Finally, the first one comes from the lemma 6.4 above and proposition 3.3 in [15], which states that for all $u, v \in H^{*}\left(\mathbb{R}^{n}\right)$, $c\left(u \cup v, L_{1}+L_{2}\right) \leqslant c\left(u, L_{1}\right)+c\left(v, L_{2}\right)$, where $L_{1}+L_{2}=\left\{\left(q, p_{1}+p_{2}\right) \mid\left(q, p_{1}\right) \in\right.$ $\left.L_{1},\left(q, p_{2}\right) \in L_{2}\right\}$.

Indeed, for $u=v=1_{(t, x)}, L_{1}=\left.\left(L_{H}-L_{K}\right)\right|_{(t, x)}$ and $L_{2}=\left.L_{K}\right|_{(t, x)}$, we get $c\left(1_{(t, x)},\left.L_{H}\right|_{(t, x)}\right)-c\left(1_{(t, x)},\left.L_{K}\right|_{(t, x)}\right) \leqslant-c\left(1_{(t, x)},\left.\left(L_{H}-L_{K}\right)\right|_{(t, x)}\right)$. Then, lemma 6.4 gives $-c\left(1_{(t, x)},\left.\left(L_{H}-L_{K}\right)\right|_{(t, x)}\right) \leqslant \gamma\left(L_{H}-L_{K}\right)=\gamma\left(L_{H}, L_{K}\right)$. By exchanging $H$ and $K$ and taking the supremum over $(t, x)$, we obtain $\| u_{L_{H}}-$ $u_{L_{K}} \|_{C^{0}} \leqslant \gamma\left(L_{H}, L_{K}\right)$ as required. 
Remark And Question. - Joukovskaia proved in [7] that for Hamiltonian functions that are convex in $p$, variational solutions of $(H J)$ coincide with viscosity solutions (These are a notion of weak solution introduced by Crandall and Lions in [2] that has shown its efficiency in a lot of domains of applications including optimal control and differential games, front propagation problems, finance, image theory....). We are tempted to use it together with some convergence result on viscosity solutions, to prove that our generalized variational solution is a viscosity solution. This would give another interpretation of our notion of solution, and since our solution is continuous, it would also give a continuity result on viscosity solutions.

However, since we developed our theory in the context of compactly supported Hamiltonians, we cannot reason on Hamiltonian functions convex in $p$. That leads us to our question : Can one define a completion with similar properties for a class of Hamiltonian functions convex in $p$ ?

\section{Appendix A \\ Proof of inequalities}

In this appendix we prove proposition 2.13 and lemma 2.7. All those inequalities are based on the reduction inequality stated in proposition A.1.

A.1. Inequality between $\tilde{\gamma}$ and $\gamma$. - We first prove the inequality $\gamma \geqslant \tilde{\gamma}$.

Let $\varphi$ be a Hamiltonian diffeomorphism, and $L \in \mathcal{L}$. We wish to show that $\gamma(\varphi(L)-L) \leqslant \gamma(\varphi)$. If we denote by $N$ the zero section of $\mathbb{R}^{2 n}=T^{*} \mathbb{R}^{n}$, there exists a Hamiltonian isotopy $\psi^{t}$ such that $L=\psi^{1}(N)$. Therefore, we just need to prove $\gamma(\varphi(N)) \leqslant \gamma(\varphi)$. Indeed, if we assume this inequality, then $\gamma(\varphi(L)-L)=\gamma\left(\varphi \circ \psi^{1}(N)-\psi^{1}(N)\right)=\gamma\left(\psi^{-1} \circ \varphi \circ \psi^{1}(N)-N\right)$, using formula (2.1) in [1]. Then, by assumption we get $\gamma(\varphi(L)-L) \leqslant \gamma\left(\psi^{-1} \circ \varphi \circ \psi^{1}\right)=\gamma(\varphi)$.

Let us prove now that $\gamma(\varphi(N)) \leqslant \gamma(\varphi)$. We denote by $\Delta_{p}$ the diagonal in $\mathbb{R}^{p} \times \mathbb{R}^{p}$, and by $\Phi$ the symplectic identification $\overline{\mathbb{R}^{2 n}} \times \mathbb{R}^{2 n} \rightarrow T^{*} \Delta_{2 n}$. Recall that $\widetilde{\Gamma_{\varphi}}$ is by definition the image of the graph $\Gamma_{\varphi}$ of $\varphi$. Clearly, $\varphi(N)$ is identified to the symplectic reduction of $N \times \Gamma_{\varphi} \subset \mathbb{R}^{6 n}$ by the coisotropic linear subspace $\Delta_{2 n} \times \mathbb{R}^{2 n}$. It is therefore identified to the reduction of $N \times \widetilde{\Gamma_{\varphi}}$ by $W=\left(I d_{\mathbb{R}^{2 n}} \times \Phi\right)\left(\Delta_{2 n} \times \mathbb{R}^{2 n}\right)$. One can easily show that for all $L \in \mathcal{L}$, $\gamma(N \times L)=\gamma(L)$. In particular, $\gamma(\varphi)=\gamma\left(N \times \widetilde{\Gamma_{\varphi}}\right)$, and the proof will be achieved if we prove the following proposition.

Proposition A.1 (Reduction Inequality). - For every Lagrangian submanifold $L$ in $\mathbb{R}^{2 n}$ and every linear coisotropic subspace $W$ of $\mathbb{R}^{2 n}$, we have $\gamma(L) \geqslant$ $\gamma\left(L_{W}\right)$, where $L_{W}$ denotes the image of $L$ by reduction by $W$.

We first prove the following lemma. 
LEMMA A.2. - Let $L$ be a Lagrangian submanifold in a cotangent bundle of the form $T^{*} M=T^{*} B \times \mathbb{R}^{2 k}$. Consider the two coisotropic submanifolds $X=$ $T^{*} B \times\left\{x_{0}\right\} \times \mathbb{R}^{n}$ and $Y=T^{*} B \times \mathbb{R}^{n} \times\{0\}$. Denote by $L_{X}$ and $L_{Y}$ the reductions of $L$ by respectively $X$ and $Y$. Then

$$
\begin{gathered}
c\left(1, L_{X}\right) \geqslant c(1, L)=c\left(1, L_{Y}\right), \\
c\left(\mu_{B}, L_{X}\right) \leqslant c\left(\mu_{M}, L\right)=c\left(\mu_{B}, L_{Y}\right) .
\end{gathered}
$$

Proof. - We start the proof by showing that $c\left(1, L_{X}\right) \geqslant c(1, L)$. Let us fix $\lambda \in \mathbb{R}$ and consider the inclusion $i: B \simeq\{0\} \times B \rightarrow M$. Let $S$ be a g.f.q.i. of $L$ defined on a bundle $\pi: E \rightarrow M$. Then the function $S_{X}=\left.S\right|_{\pi^{-1}\left(B \times\left\{x_{0}\right\}\right)}$ is a generating function for $L_{X}$. Since $S_{X}$ is a restriction of $S$, we have an inclusion of the sublevels $S_{X}^{\lambda} \subset S^{\lambda}$, which induces a morphism $i_{\lambda}: H^{*}\left(S^{\lambda}, S^{-\infty}\right) \rightarrow$ $H^{*}\left(S_{X}^{\lambda}, S_{X}^{-\infty}\right)$. The naturality of Thom isomorphism and the fact that all different inclusions commute make the following diagram commutative.

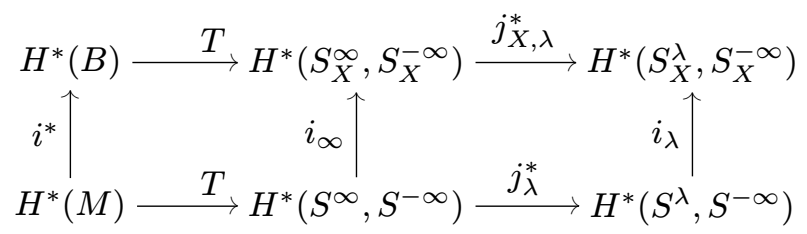

Suppose now that $j_{X, \lambda}^{*} \circ T(1) \neq 0$. Then $i_{\lambda} \circ j_{\lambda}^{*} \circ T(1)=j_{X, \lambda}^{*} \circ T \circ i^{*}(1)=$ $j_{X, \lambda}^{*} \circ T(1) \neq 0$ hence $j_{\lambda}^{*} \circ T(1) \neq 0$. That proves $c\left(1, L_{X}\right) \geqslant c(1, L)$.

In the case of $L_{Y}$, we also have an explicit generating function, constructed as follows. Since $\mathbb{R}^{k}$ is contractible we can suppose that the fibers of $\pi$ do not depend on the second coordinate of $M$. Denote by $i: B \simeq B \times\{0\} \rightarrow$ $E$ the inclusion and by $\tau: B \times \mathbb{R}^{k} \rightarrow B$ the trivial bundle of rank $k$ over $B$. Consider the vector bundle over $B, \rho=\tau \oplus i^{*} \pi$ whose total space is $F=\pi^{-1}(B \times\{0\}) \times \mathbb{R}^{n}$. Then, the function $S_{Y}$, defined for all $v \in B$ and $(x, \xi) \in \rho^{-1}(v)$ by $S_{Y}(v ; x, \xi)=S(v, x ; \xi)$ is a g.f.q.i for $L_{Y}$. The map $f: E \rightarrow$ $F,(v, x ; \xi) \mapsto(v ; x, \xi)$ is a diffeomorphism and satisfies $S_{Y} \circ f=S$. Therefore, we have $S_{Y}^{\lambda}=f\left(S^{\lambda}\right)$, an isomorphism $H^{*}\left(S^{\lambda}, S^{-\infty}\right) \simeq H^{*}\left(S_{Y}^{\lambda}, S_{Y}^{-\infty}\right)$ and a commutative diagram

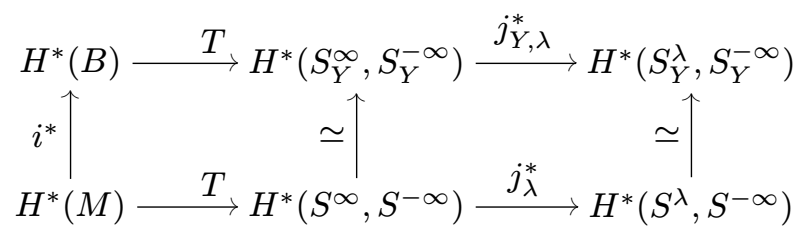

TOME $136-2008-\mathrm{N}^{\circ} 3$ 
The previous argument gives $c\left(1, L_{Y}\right) \geqslant c(1, L)$. The reverse inequality is obtained from the same diagram with the inclusion $i$ replaced by the projection $p: M \rightarrow B$ (which reverses vertical arrows).

Finally, $c\left(\mu_{B}, L_{N}\right) \leqslant c\left(\mu_{M}, L\right)=c\left(\mu_{B}, L_{Y}\right)$ is obtained from $c\left(1, L_{X}\right) \geqslant$ $c(1, L)=c\left(1, L_{Y}\right)$ by Poincaré duality, by noticing that $\overline{L_{X}}=\bar{L}_{X}$ and $\overline{L_{Y}}=$ $\bar{L}_{Y}$.

Lemma A.3. - Let $W$ be a coisotropic linear subspace of $\mathbb{R}^{2 n}$. Denote by $N$ the zero section of $\mathbb{R}^{2 n}=T^{*} \mathbb{R}^{n}$. Then there exists a decomposition in linear isotropic subspaces $\mathbb{R}^{2 n}=N_{1} \oplus V_{1} \oplus N_{2} \oplus V_{2} \oplus N_{3} \oplus V_{3}$, where $N=$ $N_{1} \oplus N_{2} \oplus N_{3}$ and each $N_{i} \oplus V_{i}, i=1,2,3$ is a symplectic subspace, such that $W=N_{1} \oplus V_{1} \oplus N_{2} \oplus V_{3}$.

Proof. - Let us first recall that if $W$ is coisotropic with symplectic orthogonal $W^{\omega} \subset W$, any subspace $F$ such that $F \oplus W^{\omega}=W$ is symplectic. Indeed, since $F \subset W, F \cap F^{\omega}=F \cap F^{\omega} \cap W=F \cap\left(F \oplus W^{\omega}\right)^{\omega}=F \cap W^{\omega}=\{0\}$.

If there exists a decomposition as in the lemma, then $W^{\omega}=N_{2} \oplus V_{3}$. Therefore we set $N_{2}=W^{\omega} \cap N$. Then, we define $N_{1}$ as one complementary of $N_{2}$ in $W \cap N$, and $F_{1}$ as one complementary of $W^{\omega}$ in $W$, containing $N_{1}$. By the above remark, $F_{1}$ is symplectic, and we can choose $V_{1}$ as one Lagrangian complementary of $N_{1}$ in $F_{1}$.

Then, we define $V_{3}$ as a complementary of $N_{2}$ in $W^{\omega}$. Since $W \cap N=N_{1} \oplus N_{2}$, $V_{3} \cap N=0$, and we can define $N_{3}$ as a complementary of $N_{1} \oplus N_{2}$ in $N$. Then, $F_{3}=N_{3} \oplus V_{3}$ is symplectic since it is a complementary of $\left(N_{1} \oplus N_{2} \oplus F_{3}\right)^{\omega}$ in $N_{1} \oplus N_{2} \oplus F_{3}$.

Finally, we define $F_{2}$ as a complementary of $F_{1} \oplus F_{3}$ in $\mathbb{R}^{2 n}$. Then, $F_{2}$ is symplectic for a similar reason as $F_{3}$, and we can define $V_{2}$ as a Lagrangian complementary of $N_{2}$ in $F_{2}$. The decomposition $\mathbb{R}^{2 n}=N_{1} \oplus V_{1} \oplus N_{2} \oplus V_{2} \oplus$ $N_{3} \oplus V_{3}$ satisfies all the requirements of lemma A.3.

Proof of proposition A.1. - Since the linear symplectic group acts transitively on the set of all pairs of complementary Lagrangian subspaces (see proposition 7.4 in Chapter 1 of [8]), and since the space of Lagrangian subspaces which are complementary to the zero section $N$ is path connected, there exists a symplectic isotopy $\Psi^{t}$ of $\mathbb{R}^{2 n}$ such that $\Psi^{0}=I d$ and that $\Psi^{1}$ lets all the elements of $N$ invariant and maps $V$ on $V_{1} \oplus V_{2} \oplus V_{3}$. Since $\mathbb{R}^{2 n}$ is simply connected, that isotopy is Hamiltonian.

The reduction of $L$ by $W$ is identified with the reduction of $\Psi^{1}(L)$ by $\Psi^{1}(W)$. Therefore, applying twice the lemma A.3, we get $\gamma\left(L_{W}\right) \leqslant \gamma\left(\Psi^{1}(L)\right)$. But, by proposition 2.6 in [15], we have $\gamma(L)=\gamma\left(\Psi^{1}(L)\right)$. That concludes the proof of proposition A.1. 
REMARK. - Note that in the end of the previous proof, lemma A.3 also implies $c\left(1, L_{W}\right) \geqslant c(1, L)$. That will be useful in the proof of lemma 2.7.

\section{A.2. Inequalities involving the "suspended distances"}

Proof of Proposition 2.13. - We now prove the inequality $\gamma_{u}(H, K) \leqslant$ $\hat{\gamma}(H, K)$, for any $H, K$ Hamiltonian functions. It is sufficient to prove that for all Hamiltonian functions $H, K$, all $s$ in [0,1], and all $\alpha$ large enough, $\gamma\left(\phi_{K}^{-s} \phi_{H}^{s}\right) \leqslant \gamma\left(\phi_{\hat{K}}^{-s} \phi_{\hat{H}}^{s}\right)$. We will prove that the graph of $\phi_{K}^{-s} \phi_{H}^{s}$ can be obtained by reduction of the graph of $\phi_{\hat{K}}^{-s} \phi_{\hat{H}}^{s}$, and then use proposition A.1.

We denote by $\hat{\Phi}^{s}$ the flow at time $s$ of the Hamiltonian $\hat{H}:(s ; t, \tau, x) \mapsto$ $\rho_{\alpha}(\tau) \tau+\rho_{\alpha}(t) H(t ; x)$. By direct computation, we get

$$
\hat{\Phi}^{s}(t, \tau, x)=(t(s), \tau(s), x(s)),
$$

with

$$
\begin{aligned}
t(s) & =t+\int_{0}^{s}\left(\rho_{\alpha}^{\prime}(\tau(\sigma)) \tau(\sigma)+\rho_{\alpha}(\tau(\sigma)) d \sigma\right. \\
\tau(s) & =\tau-\int_{0}^{s}\left(\rho_{\alpha}^{\prime}(t(\sigma)) H\left(t(\sigma), x(\sigma)+\rho_{\alpha}(t(\sigma)) \frac{\partial H}{\partial t}(t(\sigma), x(\sigma))\right) d \sigma\right.
\end{aligned}
$$

and $x(s)$ solution of $\dot{x}(s)=\rho_{\alpha}(t(s)) X_{H}(t(s), x(s))$. If we denote $M=$ $\max \left(\left\|\rho_{\alpha}\right\|_{C^{1}},\|H\|_{C^{1}}\right)$, we see that $\tau(s) \in\left[\tau-|s| M^{2}, \tau+|s| M^{2}\right]$. Suppose $\tau \in\left[-M^{2}-2 M, M^{2}+2 M\right]$ and $\alpha$ is large enough, then $\rho_{\alpha}(\tau(s))=1$ and $t(s)=t+s$. Hence $x(s)=\left(\phi_{H}\right)_{t}^{t+s}(x)$. We set

$$
\begin{aligned}
I_{H}(s, t, x) & =-\int_{0}^{s}\left(\rho_{\alpha}^{\prime}(t(\sigma)) H\left(t(\sigma), x(\sigma)+\rho_{\alpha}(t(\sigma)) \frac{\partial H}{\partial t}(t(\sigma), x(\sigma))\right) d \sigma\right. \\
& =H(t, x)-H\left(t+s, \phi_{t}^{t+s}(x)\right),
\end{aligned}
$$

and $J(s, t, x)=I_{H}(s, t, x)+I_{K}\left(-s, t+s,\left(\phi_{H}\right)_{t}^{t+s}(x)\right)$. Then, we can write the expression of the composition:

$$
\phi_{\hat{K}}^{-s} \phi_{\hat{H}}^{s}(t, \tau, x)=\left(t, \tau+J(s, t, x),\left(\phi_{K}\right)_{t}^{t-s}\left(\phi_{H}\right)_{t}^{t+s}(x)\right) .
$$

We can now compute the intersection of the graph $\Gamma_{\phi_{\hat{K}}^{-s} \phi_{\hat{H}}^{s}}$ with the set $U=[-1,1] \times \mathbb{R} \times\left[-M^{2}, M^{2}\right] \times \mathbb{R} \times \mathbb{R}^{2 n} \times \mathbb{R}^{2 n}$, and its image by the natural identification $\Psi: \mathbb{R}^{4+4 n} \rightarrow T^{*} \Delta_{2+2 n}$. We get

$$
\begin{aligned}
& \widetilde{\Gamma}_{\phi_{\hat{K}}^{-s} \phi_{\hat{H}}^{s}} \cap \Psi(U)=\left\{\left(t, J(s, t, x), \tau+\frac{1}{2} J(s, t, x), 0, z(x)\right) \mid\right. \\
& \quad(t, \tau, x) \in[0,1] \times\left[-M^{2}, M^{2}\right] \times \mathbb{R}^{2 n}, z(x) \in \widetilde{\Gamma}_{\left.\left(\phi_{K}\right)_{t}^{t-s}\left(\phi_{H}\right)_{t}^{t+s}\right\} .}
\end{aligned}
$$

Consider the coisotropic submanifold $W=\{0\} \times \mathbb{R} \times\{0\} \times \mathbb{R} \times \mathbb{R}^{4 n}$. Since $\tau+\frac{1}{2} J(s, t, x)=0$ implies $\tau \in\left[-M^{2}-2 M, M^{2}+2 M\right]$, and since $W \subset \Psi(U)$, we 
see that $\widetilde{\Gamma}_{\phi_{K}^{-s} \phi_{H}^{s}}$ is obtained from $\widetilde{\Gamma}_{\phi_{\hat{K}}^{-s} \phi_{\hat{H}}^{s}}$ by reduction by $W$. By proposition A.1, we get $\gamma\left(\widetilde{\Gamma}_{\phi_{K}^{-s} \phi_{H}^{s}}\right) \leqslant \gamma\left(\widetilde{\Gamma}_{\phi_{\hat{K}}^{-s} \phi_{\hat{H}}^{s}}\right)$ and hence $\gamma\left(\phi_{K}^{-s} \phi_{H}^{s}\right) \leqslant \gamma\left(\phi_{\hat{K}}^{-s} \phi_{\hat{H}}^{s}\right)$.

We are now going to prove $\gamma_{u}(H, K) \leqslant \check{\gamma}(H, K)$. The idea of the proof is the same as the previous one: we show that for any $s \in[0,1], \widetilde{\Gamma}_{\phi_{K}^{-s} \phi_{H}^{s}}$ is obtained by reduction of $\widetilde{\Gamma}_{\phi_{\breve{K}}{ }^{-1} \phi_{\breve{H}}}$, for $\alpha$ large enough.

Recall that by definition, $\check{H}(s ; t, \tau, x)=\rho_{\alpha}(t) t H(s t ; x)$. As above, we compute the flow : $\phi_{\breve{H}}^{s}(t, \tau, x)=(t(s), \tau(s), x(s))$, and we obtain

$$
\begin{aligned}
t(s) & =t \\
\tau(s) & =\tau+I_{H}(s, t, x)
\end{aligned}
$$

where $I_{H}(s, t, x)=\rho_{\alpha}(t) s H(s t, x(s))-\rho_{\alpha}^{\prime}(t) t \int_{0}^{s} H(\sigma t, x(\sigma)) d \sigma$ and $x(s)$ is solution of $\dot{x}(s)=\rho_{\alpha}(t) t X_{H}(s t, x(s))$. For $t \in[-1,1]$ and $\alpha \geqslant 1$, it gives $x(s)=\phi^{t s}(x)$.

Similarly as above, we set $J(s, t, x)=I_{H}(s, t, x)+I_{K}\left(-s, t+s,\left(\phi_{H}\right)^{t s}(x)\right)$, the set $U=[-1,1] \times \mathbb{R} \times \mathbb{R}^{2} \times \mathbb{R}^{2 n} \times \mathbb{R}^{2 n}$ and the identification $\Psi: \mathbb{R}^{4+4 n} \rightarrow$ $T^{*} \Delta_{2+2 n}$. The graph can be written this way:

$$
\begin{array}{r}
\widetilde{\Gamma}_{\phi_{\tilde{K}}^{-s} \phi_{\breve{H}}^{s}} \cap \Psi(U)=\left\{\left(t, J(s, t, x), \tau+\frac{1}{2} J(s, t, x), 0, z(x)\right) \mid\right. \\
\left.(t, \tau, x) \in[0,1] \times \mathbb{R} \times \mathbb{R}^{2 n}, z(x) \in \widetilde{\Gamma}_{\phi_{K}^{-s t} \phi_{H}^{s t}}\right\} .
\end{array}
$$

Now, we see that $\widetilde{\Gamma}_{\phi_{K}^{-t} \phi_{H}^{t}}$ is the reduction of $\widetilde{\Gamma}_{\phi_{\breve{K}}{ }^{-1} \phi_{\breve{H}}}$ by the coisotropic manifold $W=\{t\} \times \mathbb{R} \times\{0\} \times \mathbb{R} \times \mathbb{R}^{4 n}$. Using lemma A.2 twice, we conclude that for all $t \in[0,1], \gamma\left(\phi_{K}^{-t} \phi_{H}^{t}\right) \leqslant \gamma\left(\phi_{\breve{K}}^{-1} \phi_{\breve{H}}\right)$.

Proof of lemma 2.7. - It is sufficient to show that $c(V) \leqslant c\left(\mathbb{R}^{2} \times V\right)$ for all open subset $V \in \mathbb{R}^{2 n}$. Let $H$ be a Hamiltonian function with support in $V$. We just have to find a Hamiltonian function $K$ with support in $V \times \mathbb{R}^{2}$ satisfying the inequality $c_{+}(H) \leqslant c_{+}(K)$. If we set $K=\check{H}_{\alpha}$ for $\alpha$ large enough, $K$ has support in $\mathbb{R}^{2} \times V$, and we saw in particular in the previous proof that $\widetilde{\Gamma}_{\phi_{H}^{1}}$ is the reduction of $\widetilde{\Gamma}_{\phi_{\check{H}}}$. Therefore, by the remark that ends section A.1, we have $c_{+}(H) \leqslant c_{+}(K)$ as required.

\section{BIBLIOGRAPHY}

[1] F. Cardin \& C. Viterbo - "Commuting Hamiltonians and HamiltonJacobi multi-time equations", preprint, arXiv:math.SG/0507418, $2005 / 2007$. 
[2] M. Crandall \& P.-L. Lions - "Viscosity solutions of Hamilton-Jacobi equations", Trans. Amer. Math. Soc. 277 (1983), p. 1-42.

[3] M. Golubitsky \& V. Guillemin - Stable mappings and their singularities, Graduate texts in mathematics, vol. 14, Springer, 1973.

[4] H. Hofer - "On the topological properties of symplectic maps", Proc. Roy. Soc. Edinburgh Sect. A 115 (1990), p. 25-38.

[5] H. HOFER \& E. ZEHNDER - Symplectic invariants and hamiltonian dynamics, Birkhäuser, 1994.

[6] D. Husemoller - Fiber bundles, Springer, 1975.

[7] T. JoukovskaÏA - "Singularités de minimax et solutions faibles d'équations aux dérivées partielles", Ph.D. Thesis, université Paris 7, 1993.

[8] P. Libermann \& C.-M. MARLE - Geométrie symplectique, bases théoriques de la mécanique, tome I, Publications Mathématiques de l'Université Paris VII, 1986.

[9] Y. OH - "The group of Hamiltonian homeomorphisms and $C^{0}$ symplectic topology I", preprint, arXiv:math.SG/0402210, 2005.

[10] A. Ottolenghi \& C. Viterbo - "Solutions généralisées pour l'équation d'Hamilton-Jacobi dans le cas d'évolution.", manuscript.

[11] M. SCHWARZ - "On the action spectrum for closed symplectically aspherical manifolds", Pacific J. Math. 193 (2000), p. 419-461.

[12] J. C. SikoraV - "Sur les immersions Lagrangiennes admettant une phase génératrice globale", Compte-rendus de l'Académie des Sciences $\mathbf{3 0 2}$ (1986), p. 119-122.

[13] D. THÉRET - "A complete proof of Viterbo's uniqueness theorem on generating functions", Topology and its Applications 96 (1999), p. 246-266.

[14] C. Viterbo - "Solutions d'équations de Hamilton-Jacobi", Seminaire XEDP, Palaiseau, 1992.

[15] _ "Symplectic topology as the geometry of generating functions", Math. Annalen 292 (1992), p. 685-710. 